\title{
Synthesis and Photocatalytic Activities of Dinuclear Iridium Polyhydride Complexes bearing BINAP Ligands
}

\author{
Yuki Sofue, Kotohiro Nomura, Akiko Inagaki* \\ Department of Chemistry, Graduate School of Science, Tokyo Metropolitan University, Minami- \\ Osawa 1-1, Hachioji, Tokyo, 192-0397.
}

\begin{tabular}{|c|c|}
\hline 1. Experimental Procedures for the synthesis and characterization of 2a-c. & $S 2$ \\
\hline 2. Figure $\mathrm{S} 1 .{ }^{1} \mathrm{H}$ NMR spectrum of $\mathbf{2 a}$. & $S 4$ \\
\hline Figure S2. ${ }^{1} \mathrm{H}$ NMR spectrum of $\mathbf{2 a}$ (hydride region). & $S 4$ \\
\hline Figure S3. ${ }^{31} \mathrm{P}$ NMR spectrum of $\mathbf{2 a}$. & S5 \\
\hline 3. Figure S4. ${ }^{1} \mathrm{H}$ NMR spectrum $\mathbf{2 b}$. & S6 \\
\hline Figure S5. ${ }^{1} \mathrm{H}$ NMR spectrum of $\mathbf{2} \mathbf{b}$ (hydride region). & S6 \\
\hline Figure S6. ${ }^{31} \mathrm{P}$ NMR spectra of $\mathbf{2 b}$ & $S 7$ \\
\hline Figure S7. ${ }^{13} \mathrm{C}$ NMR spectrum of $\mathbf{2 b}$. & $S 7$ \\
\hline 4. Figure S8. ${ }^{1} \mathrm{H}$ NMR spectra of $\mathbf{2 c}$. & 58 \\
\hline Figure S9. ${ }^{1} \mathrm{H}$ NMR spectrum of $\mathbf{2 c}$ (hydride region). & $S 8$ \\
\hline Figure S10. ${ }^{31} \mathrm{P}$ NMR spectra of $\mathbf{2 c}$. & $S 9$ \\
\hline 5. X-ray Structural Determinations and ORTEP diagram of $\mathbf{2 b}$ (Figure S11). & $S 10$ \\
\hline 6. Table S1. Crystal data and structure refinement for $\mathbf{2 b}$. & S11 \\
\hline 7. Table S2. Bond lengths $[\AA]$ and angles $\left[^{\circ}\right]$ for $\mathbf{2 b}$. & $S 12$ \\
\hline 8. Figure S12. Optimized structure of $\mathbf{2 a}$. & $S 21$ \\
\hline 9. Figure S13. Frontier molecular orbitals of $\mathbf{2 a}$. & $S 22$ \\
\hline $\begin{array}{l}\text { 10. Figure } \mathrm{S} 14 \text {. (a) Addition of 1-hexene ( } 30 \text { eq.) and } \mathrm{H}_{2}(1 \mathrm{~atm}) \text { after full conversion of } \\
\text { 1-hexene after } 1^{\text {st }} \text { hydrogenation of } 1 \text {-hexene under irradiation }(395 \mathrm{~nm}) \text { by catalyst } \\
\text { 2a. (b) Time course for the catalytic hydrogenation of } 1 \text {-hexene catalyzed by } \mathbf{2 a} \\
\text { under repeated on }(395 \mathrm{~nm}) / \text { off (dark) cycles. }\end{array}$ & $S 23$ \\
\hline $\begin{array}{l}\text { 11. Figure S15. }{ }^{1} \mathrm{H} \text { NMR spectra of } \mathbf{2 a} \text { in the H-D exchange reaction under dark } \\
\text { condition. }\end{array}$ & $S 24$ \\
\hline $\begin{array}{l}\text { 12. Figure S16. }{ }^{31} \mathrm{P} \text { NMR spectra of } \mathbf{2 a} \text { in the } \mathrm{H}-\mathrm{D} \text { exchange reaction under dark } \\
\text { condition. }\end{array}$ & $S 24$ \\
\hline $\begin{array}{l}\text { 13. Figure S17. Time course of }{ }^{1} \mathrm{H} \text { NMR spectra of } \mathbf{2 a} \text { in the H-D exchange reaction } \\
\text { under irradiation }(395 \mathrm{~nm}) \text {. }\end{array}$ & $S 25$ \\
\hline $\begin{array}{l}\text { 14. Figure S18. Time course of }{ }^{31} \mathrm{P} \text { NMR spectra of } 2 \mathbf{a} \text { in the H-D exchange reaction } \\
\text { under irradiation }(395 \mathrm{~nm}) \text {. }\end{array}$ & $S 25$ \\
\hline $\begin{array}{l}\text { 15. Scheme S1. Plausible reaction mechanism of complex 2a-c with 1-hexene under } \\
\text { photoirradiation. }\end{array}$ & $S 26$ \\
\hline
\end{tabular}




\section{Experimental Procedures}

General. All experiments were carried out under a nitrogen atmosphere using standard Schlenk techniques. All solvents were treated with appropriate drying agents, distilled, and stored under nitrogen atmosphere. All the chemicals were purchased from common commercial suppliers. ${ }^{1} \mathrm{H}$ (400 $\mathrm{MHz})$ and ${ }^{31} \mathrm{P}(162 \mathrm{MHz})$ spectra were acquired on a JEOL JNM-AL400 FT-NMR spectrometers. ${ }^{13} \mathrm{C}$ NMR spectra were acquired on a Bruker AVANCE III 600 FT-NMR spectrometers. Solvents for NMR measurements $\left(\mathrm{CD}_{3} \mathrm{OD},\left(\mathrm{CD}_{3}\right)_{2} \mathrm{CO} \mathrm{CD} \mathrm{Cl}_{2}\right)$ were dried over molecular sieves, degassed, and stored under $\mathrm{N}_{2}$ atmosphere. UV-vis absorption was obtained on a JASCO V-670 spectrometer, respectively.

Computational Details. DFT calculations were performed using the Gaussian-16 Revision A.03 quantum chemistry program package ${ }^{\mathrm{i}}$ at the B3LYP/LanL2DZ level ${ }^{\mathrm{i}, \mathrm{iii}}$. We used the LanL2DZ pseudopotential for Ir, 6-31G(d) $)^{\text {iv }}$ split-valence basis set for $\mathrm{P}$, and $3-21 \mathrm{G}^{\mathrm{v}}$ for $\mathrm{C}$ and $\mathrm{H}$. The orbital energies were determined by using minimized singlet geometries to approximate the ground state.

\section{Preparation and spectral data of $1 \mathrm{a}$.}

To an acetone $(6.5 \mathrm{~mL})$ solution of $\left[\operatorname{Ir}(\operatorname{cod})_{2}\right] \mathrm{BF}_{4}(0.14 \mathrm{~g}, 0.30 \mathrm{mmol})$, a solution of BINAP $\left(2,2^{\prime}-\right.$ bis(diphenylphosphino)-1,1'-binaphthyl) $(0.19 \mathrm{~g}, 0.30 \mathrm{mmol})$ in THF $(5 \mathrm{~mL})$ was added. After stirring for $1.5 \mathrm{~h}$, the red-purple solution was concentrated under vacuum and $\mathrm{Et}_{2} \mathrm{O}$ was added for recrystallization. 1a was obtained as red-purple crystals $(0.28 \mathrm{~g}, 0.29 \mathrm{mmol}, 96 \%)$. Complexes $1 \mathbf{b}$ (85\%) and $\mathbf{1 c}(79 \%)$ were synthesized in the same way as $\mathbf{1 a}$.

\section{Preparation of 2a, $2 \mathrm{~b}$ and $2 \mathrm{c}$.}

A methanol $(1.5 \mathrm{~mL})$ solution of $1 \mathrm{a}(42.3 \mathrm{mg}, 0.042 \mathrm{mmol})$ was degassed by freeze-pump-thaw method, and then $1 \mathrm{~atm} \mathrm{H}_{2}$ gas was introduced. After stirring the solution for $3 \mathrm{~h}$, the solution became cloudy, and $\mathrm{Et}_{2} \mathrm{O}$ was added to isolate $\mathbf{2 a}$ as a yellow powder $(31.2 \mathrm{mg}, 0.0181 \mathrm{mmol}, 85 \%)$. Other related compounds $\mathbf{2 b}(74 \%)$ and $\mathbf{2 c}(85 \%)$ were synthesized by the same procedure.

Complex 2a.

${ }^{1} \mathrm{H}$ NMR (400 MHz, acetone- $\left.d_{6}, \delta / \mathrm{ppm}\right)$ 8.33-5.87 (m, $64 \mathrm{H}, \mathrm{Ph}+$ naphthyl), -6.50 (d, $J=8.0 \mathrm{~Hz}, 2$ H, Ir-H), -8.48 (t, $J=6.5$ Hz, 1 H, Ir-H), -19.61 (brs, 2 H, Ir-H).

${ }^{31} \mathrm{P}\left\{{ }^{1} \mathrm{H}\right\}$ NMR (162 MHz, acetone- $\left.d_{6}, \delta / \mathrm{ppm}\right) 27.9,19.7$ (br).

Anal. Calcd. for $\mathrm{C}_{88} \mathrm{H}_{69} \mathrm{Ir}_{2} \mathrm{P}_{4} \mathrm{SbF}_{6} \cdot 1.6 \mathrm{CH}_{2} \mathrm{Cl}_{2}: \mathrm{C}, 53.64 ; \mathrm{H}, 3.63$. Found: C, 53.24; H, 4.03.

\section{Complex 2b.}

${ }^{1} \mathrm{H}$ NMR (400 MHz, acetone- $\left.d_{6}, \delta / \mathrm{ppm}\right)$ 8.20-5.60 (m, $56 \mathrm{H}$, naphthyl), 2.56 (s, $3 \mathrm{H}, \mathrm{C}_{6} \mathrm{H}_{4}-\mathrm{CH}_{3}$ ), $2.43\left(\mathrm{~s}, 3 \mathrm{H}, \mathrm{C}_{6} \mathrm{H}_{4}-\mathrm{CH}_{3}\right), 1.91$ (s, $\left.3 \mathrm{H}, \mathrm{C}_{6} \mathrm{H}_{4}-\mathrm{CH}_{3}\right), 1.67$ (s, $\left.3 \mathrm{H}, \mathrm{C}_{6} \mathrm{H}_{4}-\mathrm{CH}_{3}\right),-6.61(\mathrm{~d}, J=7.9 \mathrm{~Hz}, 2 \mathrm{H}$, 
Ir-H), -8.65 (t, $J=6.4 \mathrm{~Hz}, 1 \mathrm{H}$, Ir-H), -19.56 (brs, $2 \mathrm{H}, \mathrm{Ir}-\mathrm{H})$

${ }^{31} \mathrm{P}\left\{{ }^{1} \mathrm{H}\right\}$ NMR (162 MHz, acetone- $\left.d_{6}, \delta / \mathrm{ppm}\right) 26.1,17.8$ (br).

${ }^{13} \mathrm{C}$ NMR (150 MHz, acetone- $\left.d_{6}, \delta / \mathrm{ppm}\right) 141.7,141.3,140.1,139.4,138.1,137.6,136.6,135.8$, 133.8, 133.6, 133.0, 132.8, 129.1, 128.6, 128.0, 127.6, 127.1, 126.9, 126.4, 126.2, 125.7, 124.6, 123.7, $20.4\left(\mathrm{CH}_{3}\right), 20.1\left(\mathrm{CH}_{3}\right)$.

Anal. Calcd. for $\mathrm{C}_{96} \mathrm{H}_{85} \mathrm{Ir}_{2} \mathrm{P}_{4} \mathrm{SbF}_{6} \cdot 2 \mathrm{H}_{2} \mathrm{O}: \mathrm{C}, 57.11 ; \mathrm{H}, 4.44$. Found: C, 56.88; H, 4.64.

Complex 2c.

${ }^{1} \mathrm{H} \mathrm{NMR}\left(400 \mathrm{MHz}, \mathrm{CD}_{2} \mathrm{Cl}_{2}, \delta / \mathrm{ppm}\right)$ 7.96-5.20 (m, $56 \mathrm{H}$ ), 5.86 (s, $2 \mathrm{H}$, methyl), 5.79 (s, $2 \mathrm{H}$, methyl), 5.71 (s, $2 \mathrm{H}$, methyl), 5.53 (s, $2 \mathrm{H}$, methyl), -6.61 (d, $J=7.8 \mathrm{~Hz}, 2 \mathrm{H}, \mathrm{Ir}-\mathrm{H}),-8.47$ (t, $J=6.6 \mathrm{~Hz}, 1 \mathrm{H}$, Ir-H), -20.00 (brs, $2 \mathrm{H}, \mathrm{Ir}-\mathrm{H}$ )

${ }^{31} \mathrm{P}\left\{{ }^{1} \mathrm{H}\right\}$ NMR (162 MHz, $\left.\mathrm{CD}_{2} \mathrm{Cl}_{2}, \delta / \mathrm{ppm}\right) 23.9,15.8(\mathrm{br})$.

Anal. Calcd. for $\mathrm{C}_{76} \mathrm{H}_{61} \mathrm{Ir}_{2} \mathrm{P}_{4} \mathrm{O}_{8} \mathrm{SbF}_{6} \cdot 3 \mathrm{CH}_{2} \mathrm{Cl}_{2}$ : C, 45.16; H, 3.21. Found: $\mathrm{C}, 45.22 ; \mathrm{H}, 3.27$. 


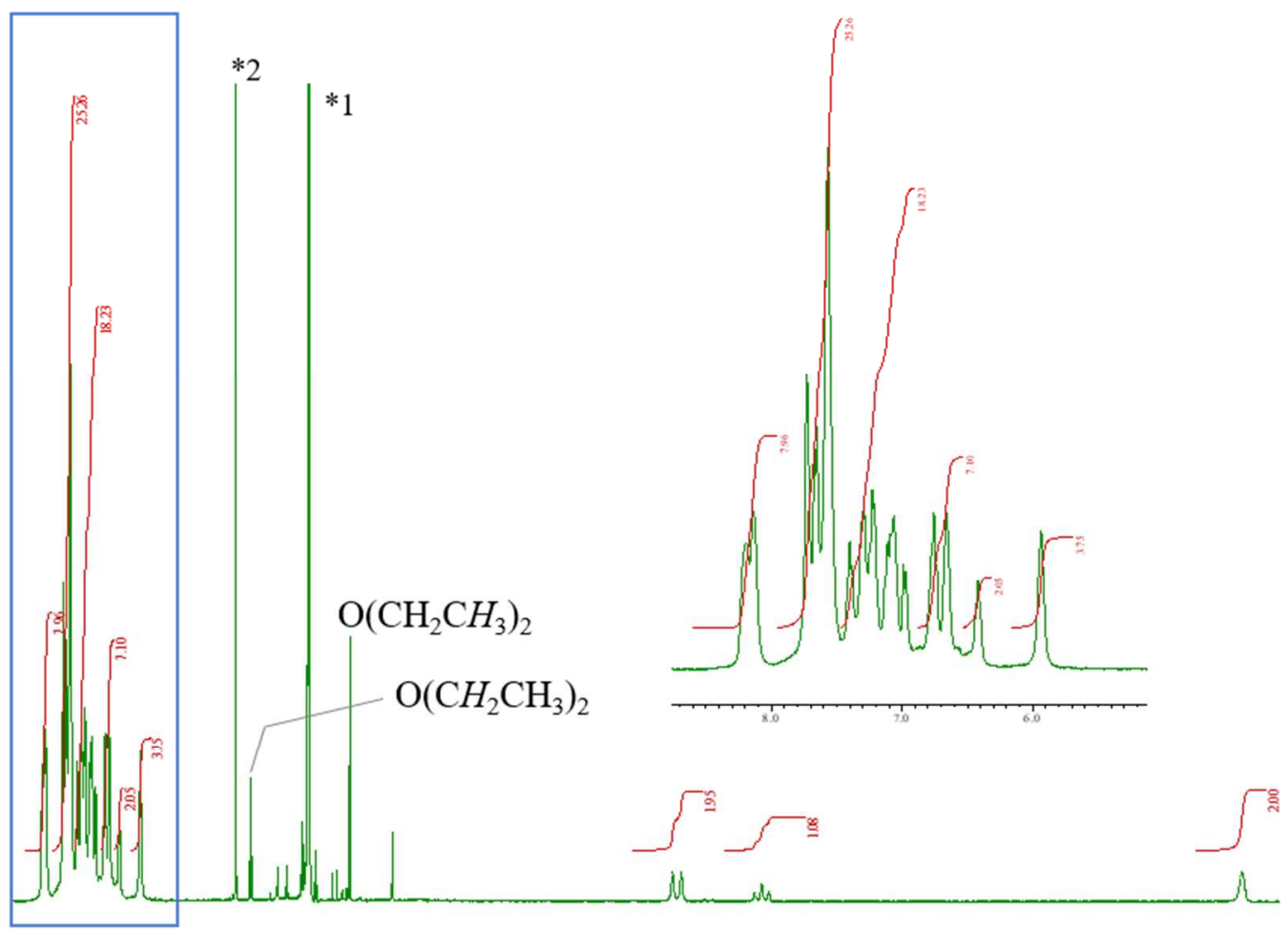

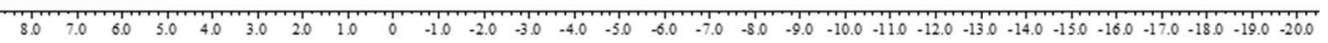

*1 residual proton signal of $\mathrm{Me}_{2} \mathrm{CO}$

*2 impurity in $\mathrm{Me}_{2} \mathrm{CO}-d_{6}$

Figure S1. ${ }^{1} \mathrm{H}$ NMR spectra of $\mathbf{2 a}\left(400 \mathrm{MHz}\right.$, acetone- $d_{6}$, r.t.)

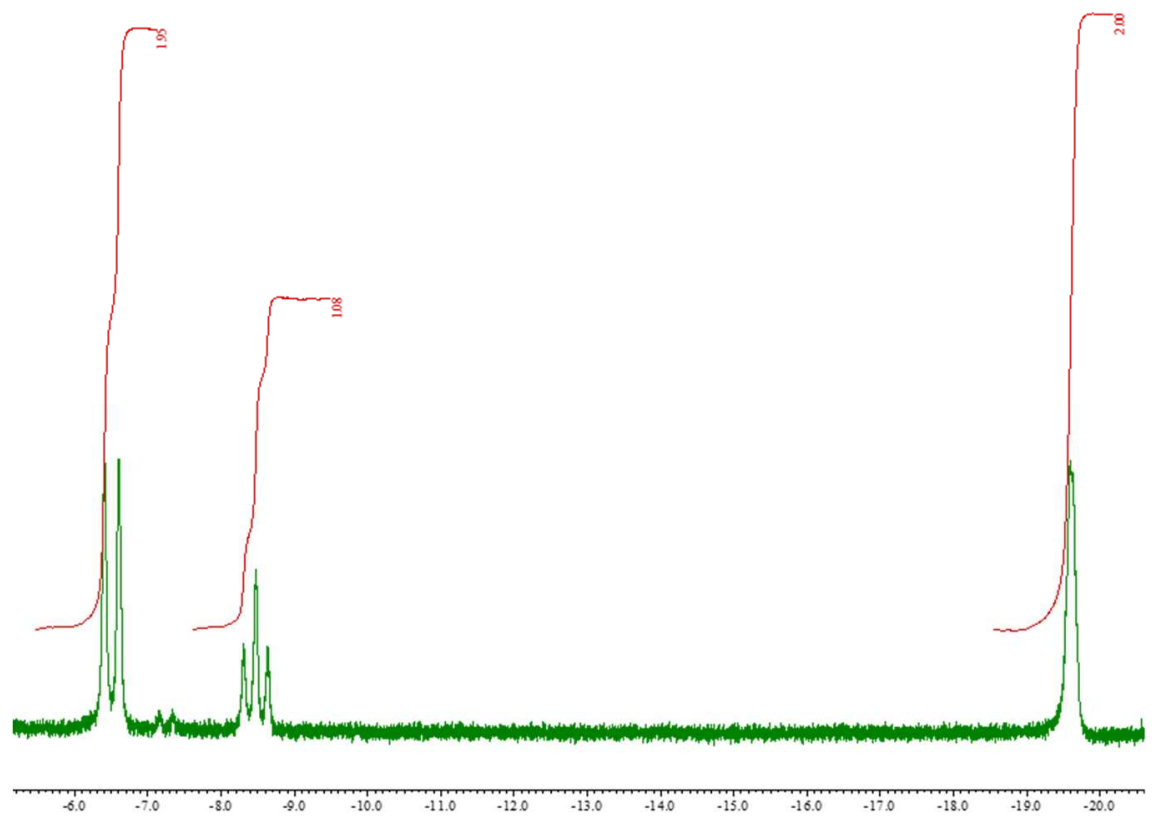

Figure S2. ${ }^{1} \mathrm{H}$ NMR spectra of hydride region of $\mathbf{2 a}\left(400 \mathrm{MHz}\right.$, acetone- $d_{6}$, r.t.). 

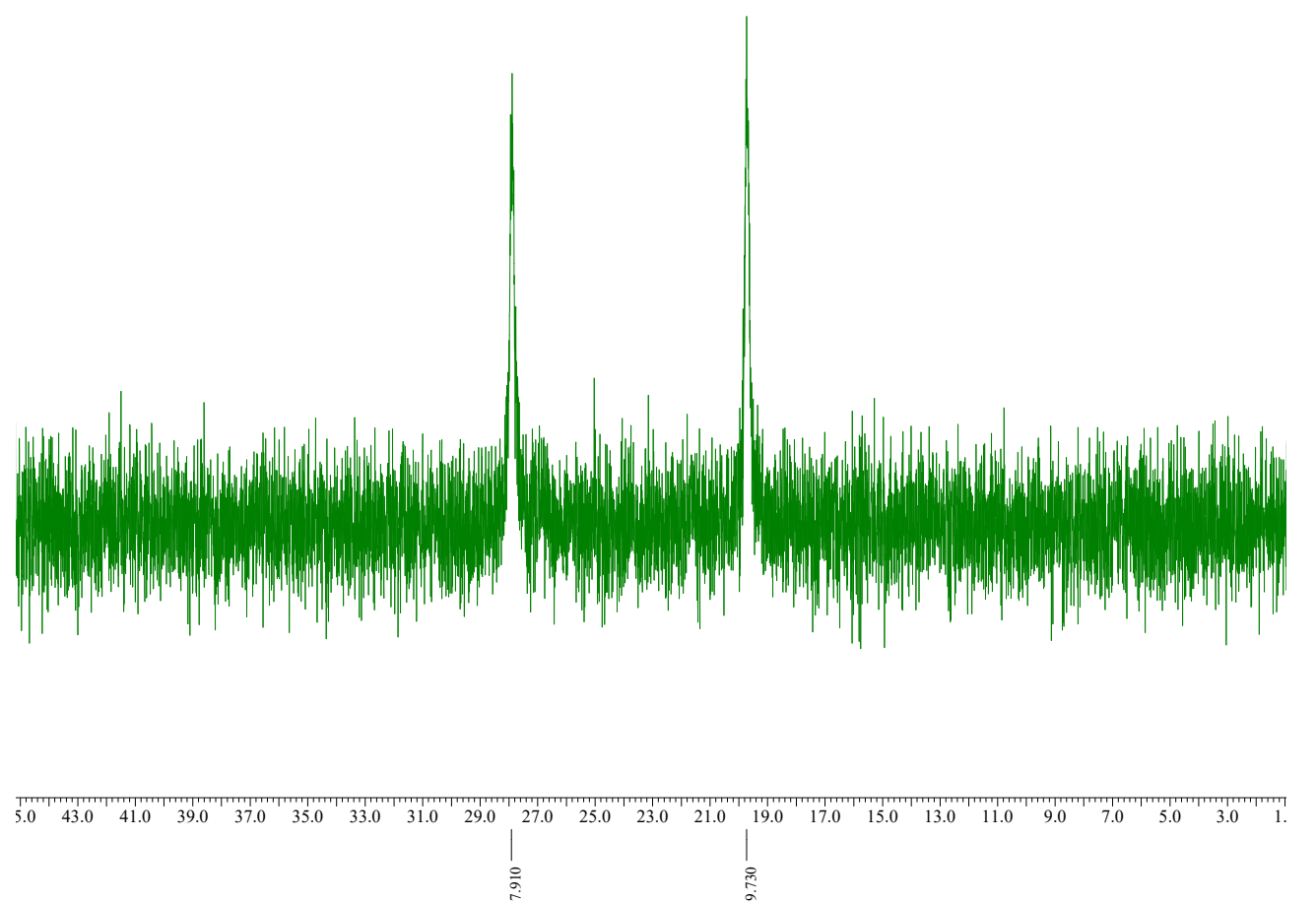

Figure S3. ${ }^{31} \mathrm{P}$ NMR spectra of 2a (161.8 MHz, acetone- $d_{6}$, r.t.). 


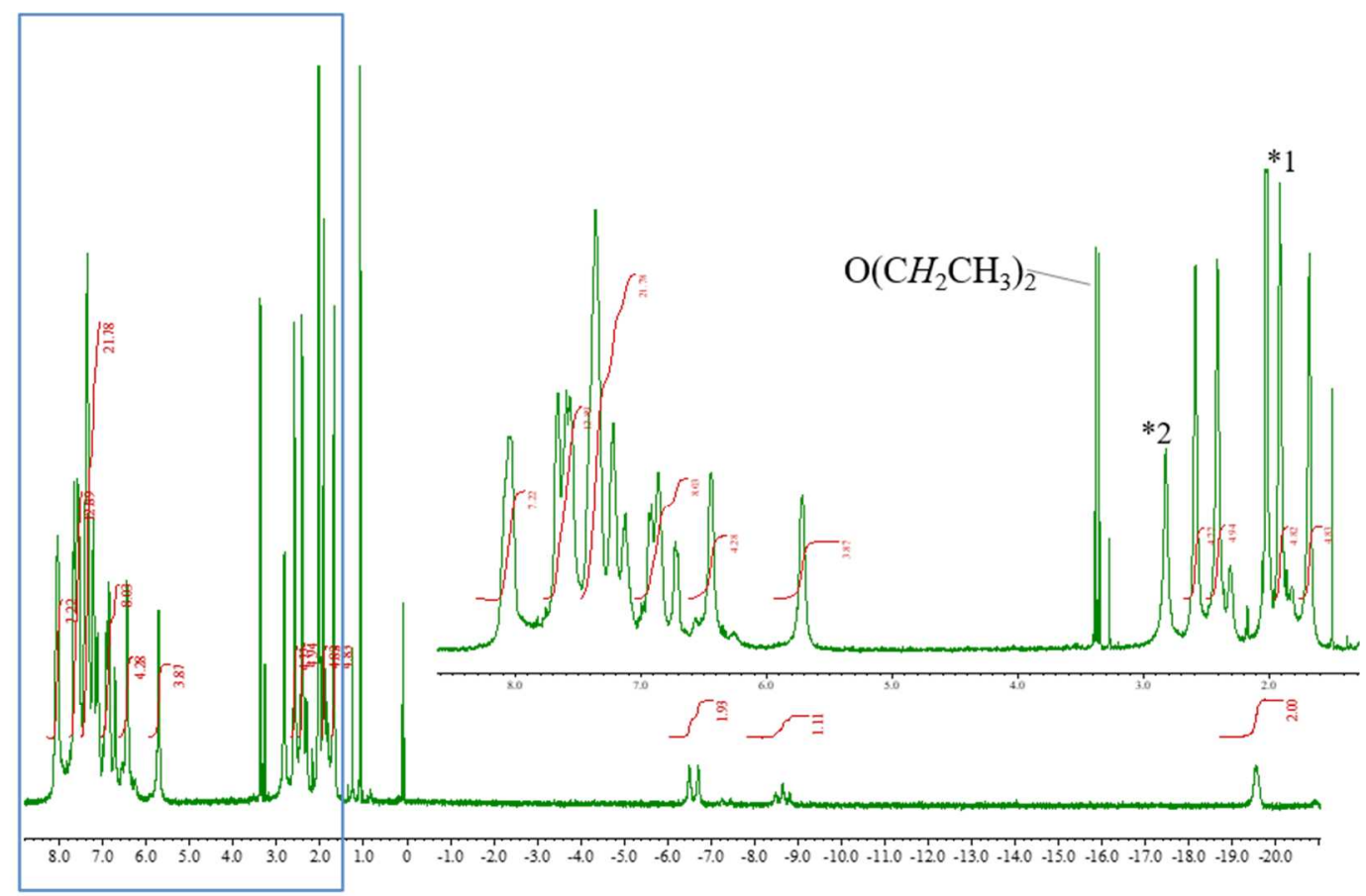

*1 residual proton signal of $\mathrm{Me}_{2} \mathrm{CO}$

$* 2 \mathrm{H}_{2} \mathrm{O}$

Figure S4. ${ }^{1} \mathrm{H}$ NMR spectra of $\mathbf{2 b}\left(400 \mathrm{MHz}\right.$, acetone- $d_{6}$, r.t.).

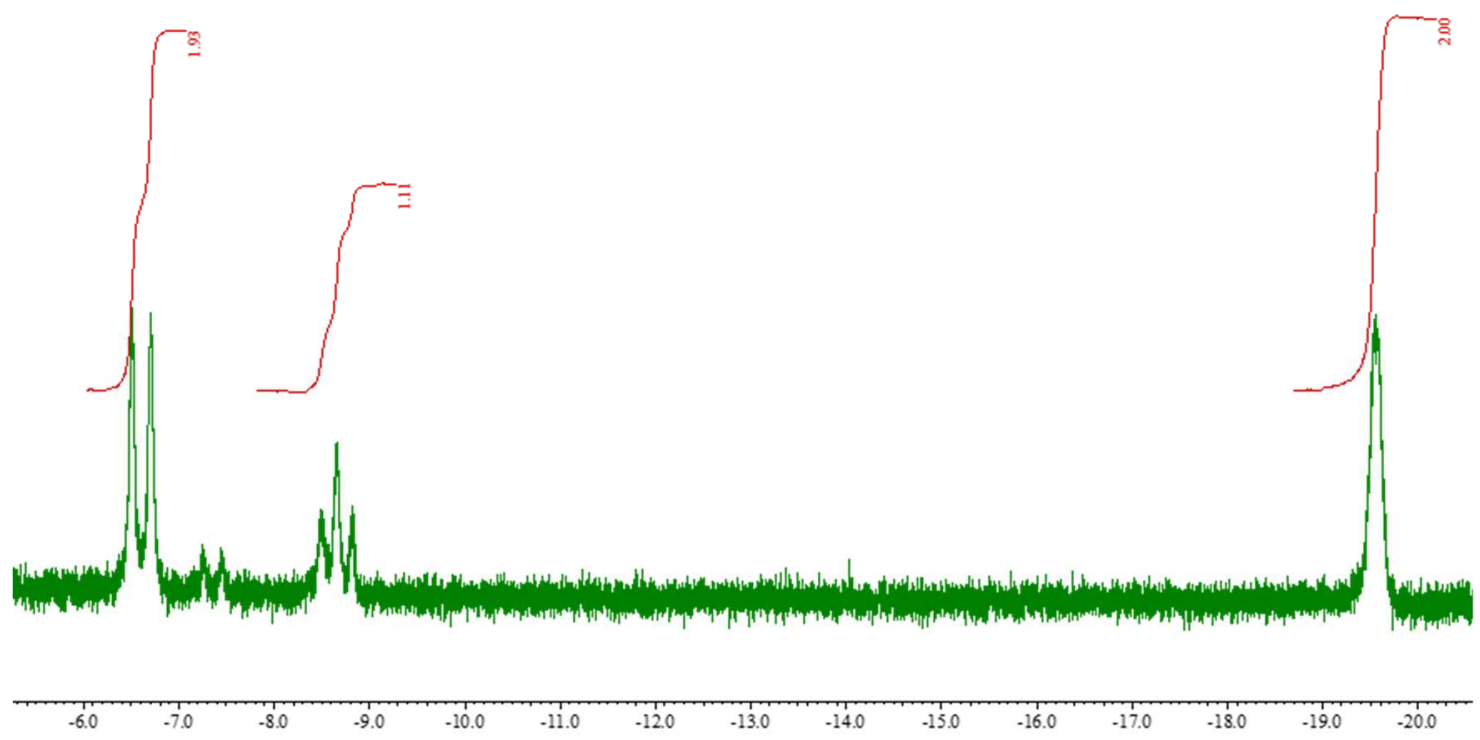

Figure S5. ${ }^{1} \mathrm{H}$ NMR spectra of hydride region of $\mathbf{2} \mathbf{b}$ ( $400 \mathrm{MHz}$, acetone- $d_{6}$, r.t.). 

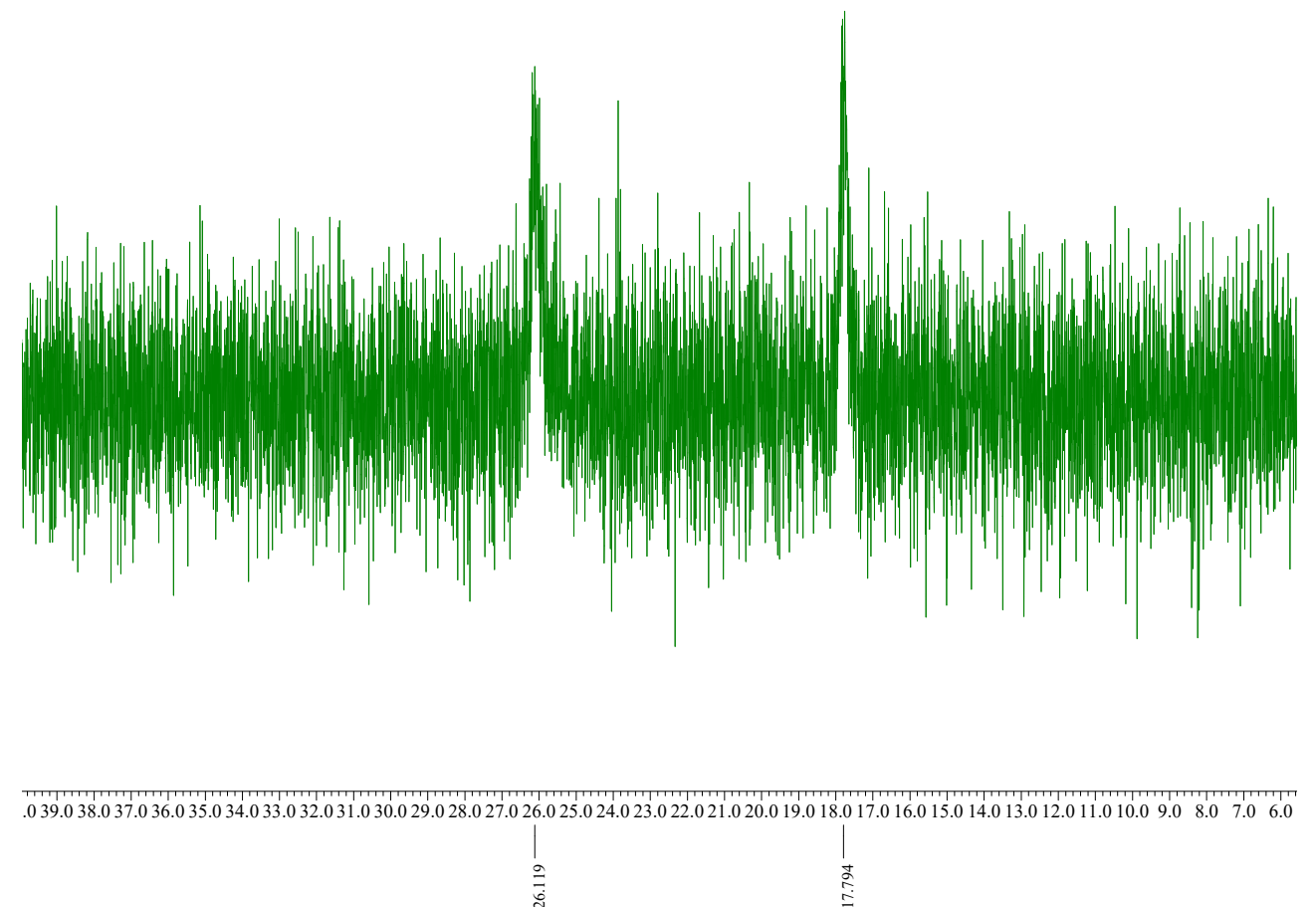

Figure S6 ${ }^{31} \mathrm{P}$ NMR spectra of $\mathbf{2 b}\left(161.8 \mathrm{MHz}\right.$, acetone- $d_{6}$, r.t.).

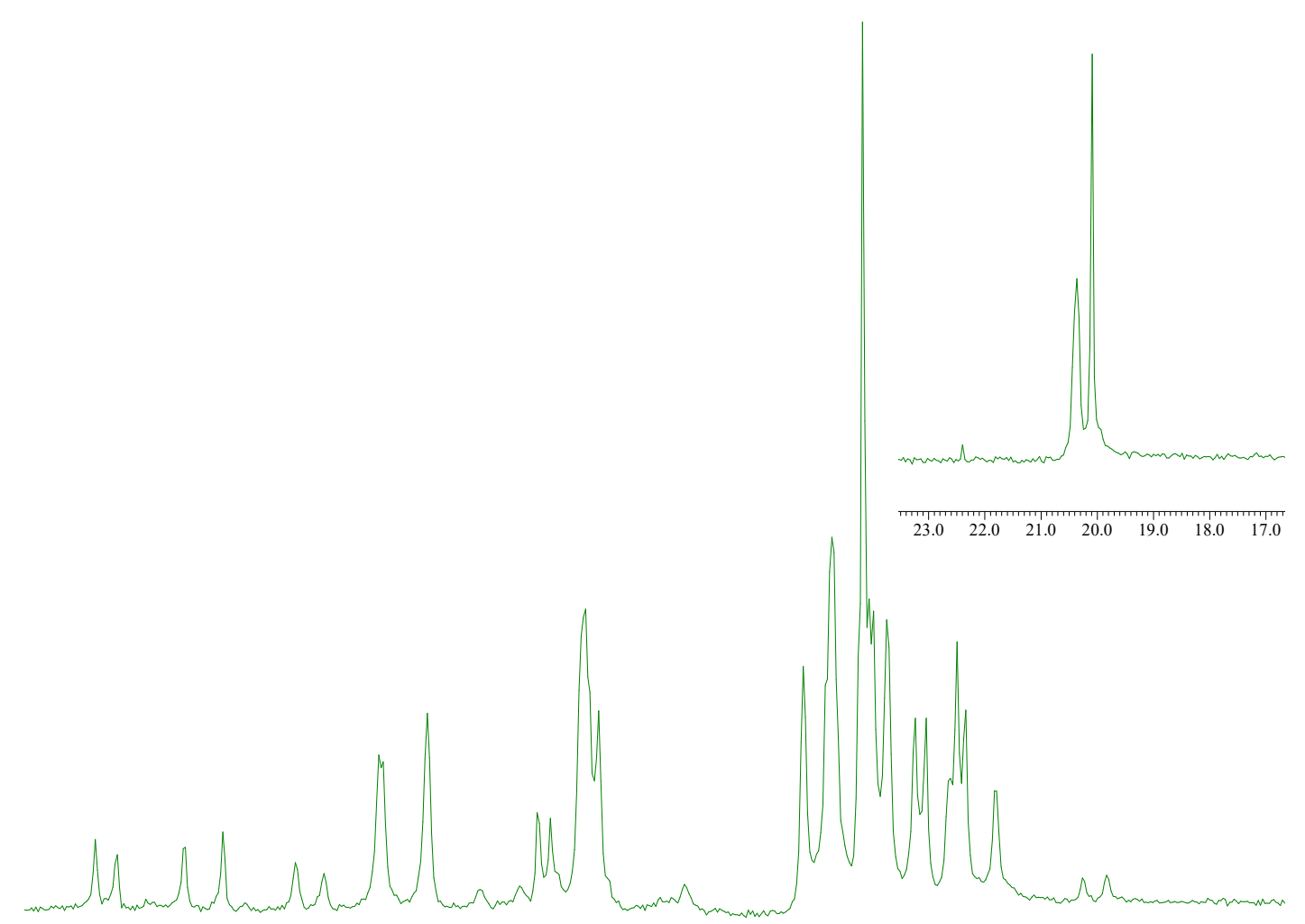

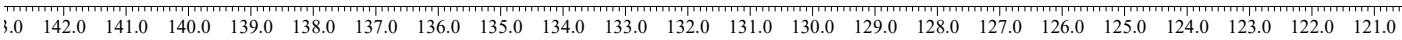

Figure S7. ${ }^{13} \mathrm{C}$ NMR spectra of $\mathbf{2 b}\left(125 \mathrm{MHz}\right.$, acetone- $d_{6}$, r.t.). 


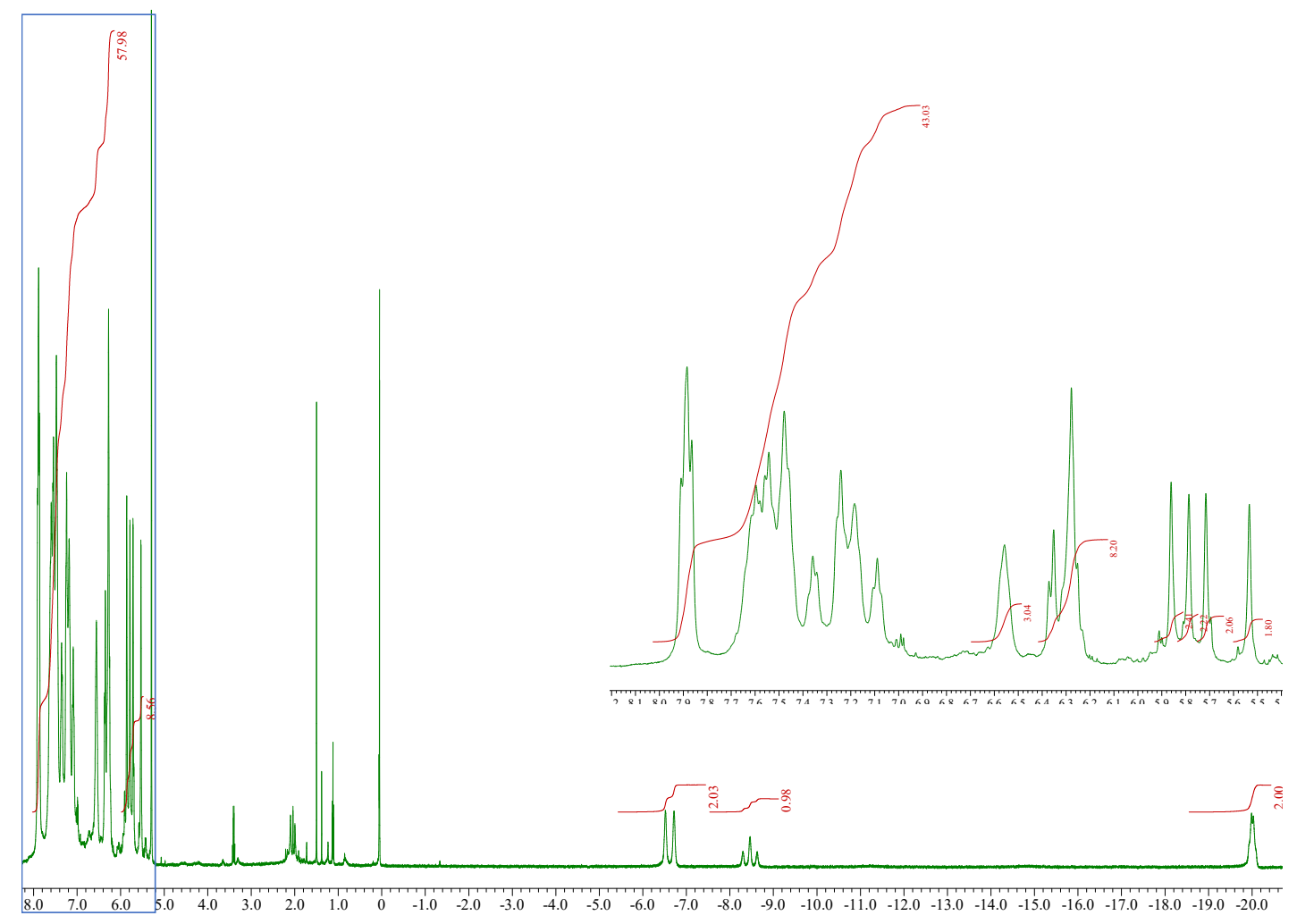

Figure S8. ${ }^{1} \mathrm{H}$ NMR spectra of $\mathbf{2 c}\left(400 \mathrm{MHz}, \mathrm{CD}_{2} \mathrm{Cl}_{2}\right.$, r.t. $)$.

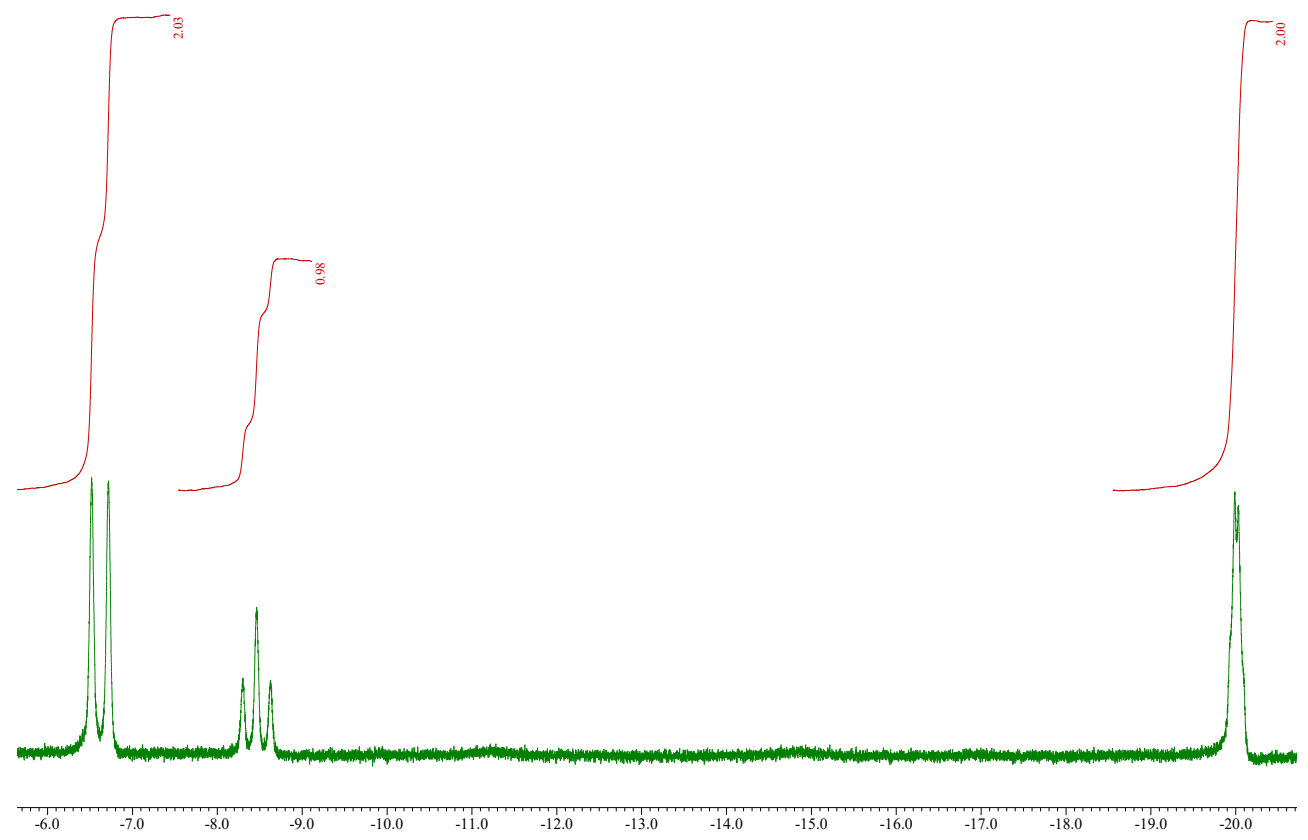

Figure S9. ${ }^{1} \mathrm{H}$ NMR spectra of hydride region of $\mathbf{2 c}\left(400 \mathrm{MHz}, \mathrm{CD}_{2} \mathrm{Cl}_{2}\right.$, r.t. $)$. 

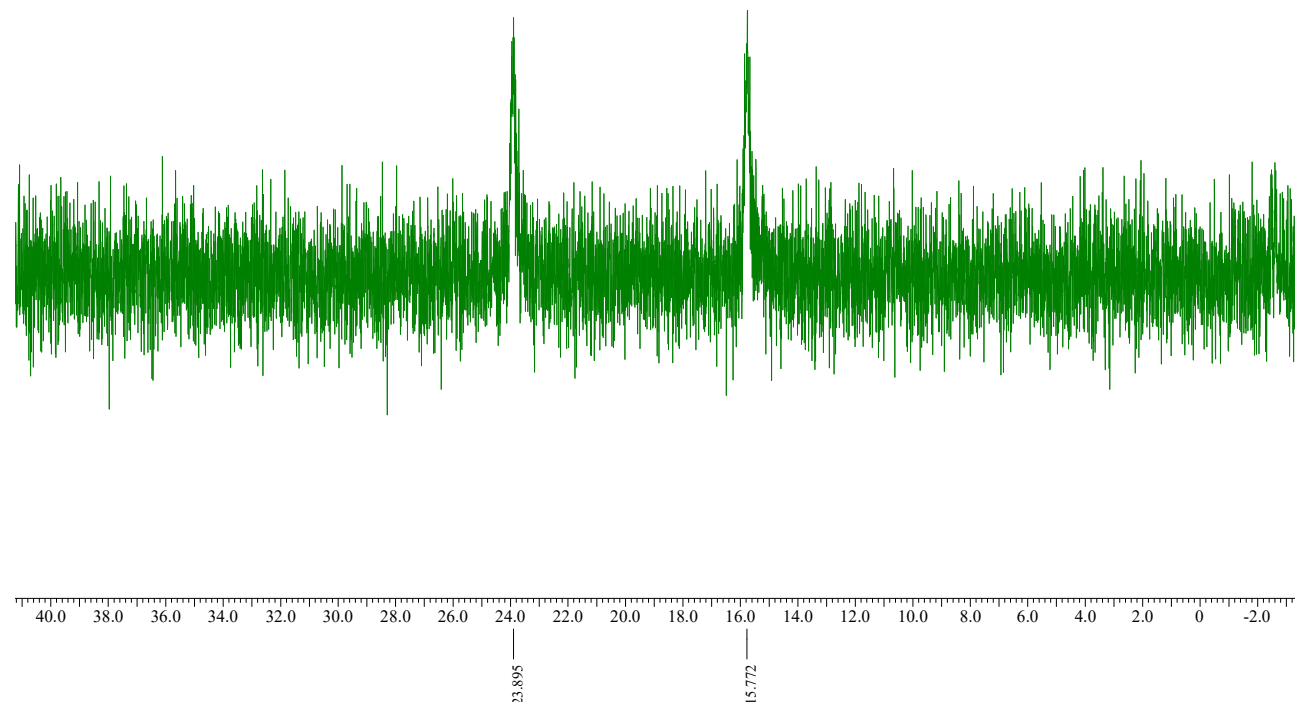

Figure S10. ${ }^{31} \mathrm{P}$ NMR spectra of $\mathbf{2 c}\left(161.8 \mathrm{MHz}, \mathrm{CD}_{2} \mathrm{Cl}_{2}\right.$, r.t. $)$. 


\section{X-ray Structural Determinations.}

The diffraction data of $\mathbf{2 b}$ were collected on a Rigaku XtaLAB P100 diffractometer with graphite monochromated $\operatorname{MoK} \alpha(\lambda=0.71073 \AA)$. The data were collected at a temperature of $-180 \pm 1{ }^{\circ} \mathrm{C}$ to a maximum $2 \theta$ value of $55.1^{\circ}$. The crystal-to-detector distance was $45.00 \mathrm{~mm}$. Readout was performed in the $0.172 \mathrm{~mm}$ pixel mode. Data were collected and processed using CrystalClear (Rigaku). ${ }^{1}$ An empirical absorption correction was applied The data were corrected for Lorentz and polarization effects.

The crystal structures were solved by direct method (SHELXS-97 ${ }^{2}$ or SHELXT ${ }^{3}$ ) and expanded using Fourier techniques, which are subsequently completed by Fourier recycling using the SHELXL 2014 program. ${ }^{3}$ Non-hydrogen atoms were refined by anisotropic displacement parameters. Crystallographic data, data collection and refinement parameters for $\mathbf{2 b}$ are listed in Table S1 and bond lengths and angles are listed in Table S2.

(1) CrystalClear: Data Collection and Processing Software, Rigaku Corporation (1998-2015). Tokyo 1968666, Japan.

(2) SHELXS Version 2013/1: Sheldrick, G. M. (2008). Acta Cryst. A64, 112-122.

(3) SHELXT Version 2014/5: Sheldrick, G. M. (2014). Acta Cryst. A70, C1437.

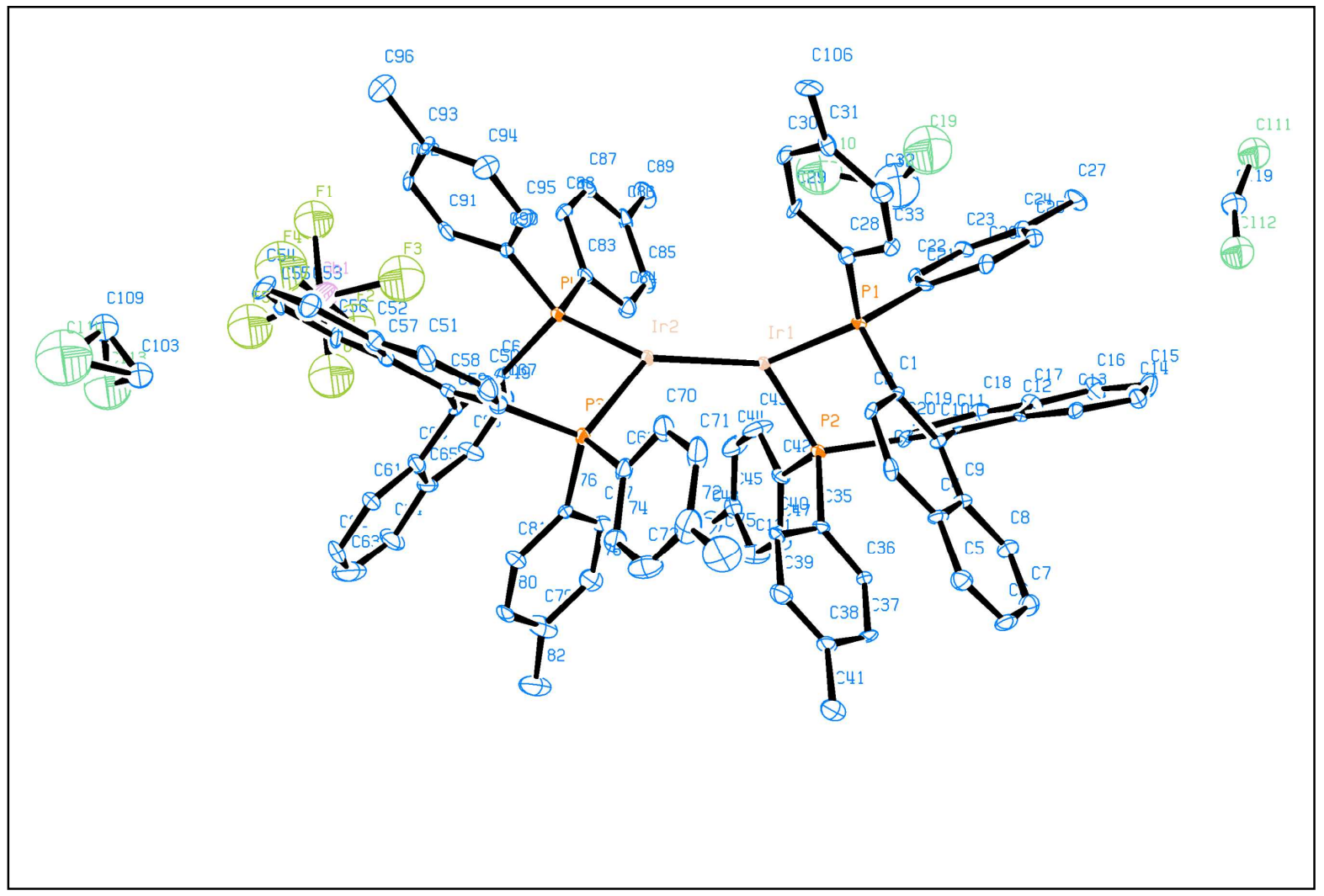

Figure S11. ORTEP diagram of $\mathbf{2 b}$. 
Table S1. Crystal data and structure refinement for $\mathbf{2 b}$.

\begin{tabular}{|c|c|}
\hline Complex & $2 \mathbf{b}$ \\
\hline Empirical formula & C99 H85 Cl6 F6 Ir2 N P4 Sb \\
\hline Formula weight & 2245.41 \\
\hline Temperature & $93(2) \mathrm{K}$ \\
\hline Wavelength & $0.71073 \AA$ \\
\hline Crystal system & Orthorhombic \\
\hline Space group & $P 22_{1} 2_{1}$ \\
\hline \multirow[t]{2}{*}{ Unit cell dimensions } & $\begin{array}{l}\mathrm{a}=11.5769(3) \AA \\
\mathrm{b}=25.8331(9) \AA \\
\mathrm{c}=30.3720(9) \AA\end{array}$ \\
\hline & $\begin{array}{l}\alpha=90^{\circ} \\
\beta=90^{\circ} \\
\gamma=90^{\circ}\end{array}$ \\
\hline Volume & $9083.3(5) \AA^{3}$ \\
\hline $\mathrm{Z}$ & 4 \\
\hline Density (calculated) & $1.642 \mathrm{Mg} / \mathrm{m}^{3}$ \\
\hline Absorption coefficient & $3.525 \mathrm{~mm}^{-1}$ \\
\hline $\mathrm{F}(000)$ & 4428 \\
\hline Crystal size & $0.600 \times 0.040 \times 0.030 \mathrm{~mm}^{3}$ \\
\hline Theta range for data collection & 2.041 to $28.201^{\circ}$ \\
\hline Index ranges & $-15<=\mathrm{h}<=13,-26<=\mathrm{k}<=34,-40<=1<=36$ \\
\hline Reflections collected & 71976 \\
\hline Independent reflections & $19834[\mathrm{R}(\mathrm{int})=0.0730]$ \\
\hline Completeness to theta $=27.50^{\circ}$ & $99.9 \%$ \\
\hline Refinement method & Full-matrix least-squares on $\mathrm{F}^{2}$ \\
\hline Data / restraints / parameters & 19834 / 0 / 995 \\
\hline Goodness-of-fit on $\mathrm{F}^{2}$ & 1.054 \\
\hline Final $R$ indices $[I>2 \operatorname{sigma}(I)]$ & $R 1=0.0661, w R 2=0.1652$ \\
\hline $\mathrm{R}$ indices (all data) & $R 1=0.0836, w R 2=0.1725$ \\
\hline Largest diff. peak and hole & 6.353 and -3.240 e. $\AA^{-3}$ \\
\hline
\end{tabular}


Table S2. Bond lengths $[\AA]$ and angles $\left[{ }^{\circ}\right]$ for $\mathbf{2 b}$.

\begin{tabular}{|c|c|}
\hline $\operatorname{Ir}(1)-\mathrm{P}(2)$ & $2.260(4)$ \\
\hline $\operatorname{Ir}(1)-\mathrm{P}(1)$ & $2.263(3)$ \\
\hline $\operatorname{Ir}(1)-\operatorname{Ir}(2)$ & $2.5173(7)$ \\
\hline $\operatorname{Ir}(2)-\mathrm{P}(3)$ & $2.246(4)$ \\
\hline $\operatorname{Ir}(2)-P(4)$ & $2.275(4)$ \\
\hline $\mathrm{P}(1)-\mathrm{C}(1)$ & $1.820(14)$ \\
\hline $\mathrm{P}(1)-\mathrm{C}(21)$ & $1.828(14)$ \\
\hline $\mathrm{P}(1)-\mathrm{C}(28)$ & $1.830(16)$ \\
\hline $\mathrm{P}(2)-\mathrm{C}(35)$ & $1.809(15)$ \\
\hline $\mathrm{P}(2)-\mathrm{C}(20)$ & $1.836(15)$ \\
\hline $\mathrm{P}(2)-\mathrm{C}(42)$ & $1.852(15)$ \\
\hline $\mathrm{P}(3)-\mathrm{C}(76)$ & $1.816(16)$ \\
\hline $\mathrm{P}(3)-\mathrm{C}(69)$ & $1.841(16)$ \\
\hline $\mathrm{P}(3)-\mathrm{C}(49)$ & $1.843(17)$ \\
\hline $\mathrm{P}(4)-\mathrm{C}(90)$ & $1.821(17)$ \\
\hline $\mathrm{P}(4)-\mathrm{C}(83)$ & $1.835(16)$ \\
\hline$P(4)-C(68)$ & $1.847(16)$ \\
\hline$C(1)-C(10)$ & $1.396(19)$ \\
\hline $\mathrm{C}(1)-\mathrm{C}(2)$ & $1.45(2)$ \\
\hline $\mathrm{C}(2)-\mathrm{C}(3)$ & $1.33(2)$ \\
\hline $\mathrm{C}(2)-\mathrm{H}(97)$ & 0.9300 \\
\hline$C(3)-C(4)$ & $1.44(2)$ \\
\hline C(3)-H(98) & 0.9300 \\
\hline$C(4)-C(9)$ & $1.38(2)$ \\
\hline $\mathrm{C}(4)-\mathrm{C}(5)$ & $1.46(2)$ \\
\hline$C(5)-C(6)$ & $1.35(2)$ \\
\hline $\mathrm{C}(5)-\mathrm{H}(99)$ & 0.9300 \\
\hline$C(6)-C(7)$ & $1.39(2)$ \\
\hline $\mathrm{C}(6)-\mathrm{H}(100)$ & 0.9300 \\
\hline$C(7)-C(8)$ & $1.34(2)$ \\
\hline $\mathrm{C}(7)-\mathrm{H}(101)$ & 0.9300 \\
\hline $\mathrm{C}(8)-\mathrm{C}(9)$ & $1.46(2)$ \\
\hline $\mathrm{C}(8)-\mathrm{H}(102)$ & 0.9300 \\
\hline $\mathrm{C}(9)-\mathrm{C}(10)$ & $1.44(2)$ \\
\hline
\end{tabular}

\begin{tabular}{|c|c|}
\hline$C(10)-C(11)$ & $1.52(2)$ \\
\hline$C(11)-C(20)$ & $1.39(2)$ \\
\hline$C(11)-C(12)$ & $1.41(2)$ \\
\hline$C(12)-C(17)$ & $1.43(2)$ \\
\hline$C(12)-C(13)$ & $1.43(2)$ \\
\hline$C(13)-C(14)$ & $1.35(2)$ \\
\hline C(13)-H(103) & 0.9300 \\
\hline$C(14)-C(15)$ & $1.40(3)$ \\
\hline C(14)-H(104) & 0.9300 \\
\hline$C(15)-C(16)$ & $1.40(3)$ \\
\hline $\mathrm{C}(15)-\mathrm{H}(105)$ & 0.9300 \\
\hline$C(16)-C(17)$ & $1.40(2)$ \\
\hline $\mathrm{C}(16)-\mathrm{H}(106)$ & 0.9300 \\
\hline $\mathrm{C}(17)-\mathrm{C}(18)$ & $1.42(2)$ \\
\hline C(18)-C(19) & $1.35(2)$ \\
\hline C(18)-H(107) & 0.9300 \\
\hline$C(19)-C(20)$ & $1.42(2)$ \\
\hline C(19)-H(108) & 0.9300 \\
\hline$C(21)-C(22)$ & $1.39(2)$ \\
\hline$C(21)-C(26)$ & $1.42(2)$ \\
\hline$C(22)-C(23)$ & $1.38(2)$ \\
\hline C(22)-H(109) & 0.9300 \\
\hline$C(23)-C(24)$ & $1.37(2)$ \\
\hline C(23)-H(110) & 0.9300 \\
\hline$C(24)-C(25)$ & $1.39(2)$ \\
\hline$C(24)-C(27)$ & $1.54(2)$ \\
\hline$C(25)-C(26)$ & $1.35(2)$ \\
\hline $\mathrm{C}(25)-\mathrm{H}(181)$ & 0.9300 \\
\hline $\mathrm{C}(26)-\mathrm{H}(112)$ & 0.9300 \\
\hline $\mathrm{C}(27)-\mathrm{H}(27)$ & 0.9600 \\
\hline $\mathrm{C}(27)-\mathrm{H}(27 \mathrm{~A})$ & 0.9600 \\
\hline $\mathrm{C}(27)-\mathrm{H}(27 \mathrm{~B})$ & 0.9600 \\
\hline$C(28)-C(33)$ & $1.40(2)$ \\
\hline$C(28)-C(29)$ & $1.40(2)$ \\
\hline
\end{tabular}




\begin{tabular}{|c|c|c|c|}
\hline$C(29)-C(30)$ & $1.38(2)$ & $\mathrm{C}(49)-\mathrm{C}(58)$ & $1.38(2)$ \\
\hline C(29)-H(113) & 0.9300 & $\mathrm{C}(49)-\mathrm{C}(50)$ & $1.43(3)$ \\
\hline$C(30)-C(31)$ & $1.40(2)$ & $C(50)-C(51)$ & $1.34(3)$ \\
\hline $\mathrm{C}(30)-\mathrm{H}(114)$ & 0.9300 & $\mathrm{C}(50)-\mathrm{H}(125)$ & 0.9300 \\
\hline $\mathrm{C}(31)-\mathrm{C}(32)$ & $1.40(2)$ & $\mathrm{C}(51)-\mathrm{C}(52)$ & $1.40(3)$ \\
\hline C(31)-C(106) & $1.50(2)$ & $\mathrm{C}(51)-\mathrm{H}(126)$ & 0.9300 \\
\hline$C(32)-C(33)$ & $1.38(2)$ & $C(52)-C(57)$ & $1.43(2)$ \\
\hline C(32)-H(115) & 0.9300 & $C(52)-C(53)$ & $1.45(2)$ \\
\hline $\mathrm{C}(33)-\mathrm{H}(116)$ & 0.9300 & $\mathrm{C}(53)-\mathrm{C}(54)$ & $1.39(3)$ \\
\hline$C(35)-C(36)$ & $1.39(2)$ & $\mathrm{C}(53)-\mathrm{H}(127)$ & 0.9300 \\
\hline $\mathrm{C}(35)-\mathrm{C}(40)$ & $1.43(2)$ & $\mathrm{C}(54)-\mathrm{C}(55)$ & $1.40(3)$ \\
\hline C(36)-C(37) & $1.39(2)$ & $\mathrm{C}(54)-\mathrm{H}(128)$ & 0.9300 \\
\hline $\mathrm{C}(36)-\mathrm{H}(117)$ & 0.9300 & $C(55)-C(56)$ & $1.35(2)$ \\
\hline C(37)-C(38) & $1.38(2)$ & $\mathrm{C}(55)-\mathrm{H}(129)$ & 0.9300 \\
\hline C(37)-H(118) & 0.9300 & $C(56)-C(57)$ & $1.44(2)$ \\
\hline C(38)-C(39) & $1.36(3)$ & $\mathrm{C}(56)-\mathrm{H}(130)$ & 0.9300 \\
\hline C(38)-C(41) & $1.55(2)$ & $C(57)-C(58)$ & $1.44(2)$ \\
\hline C(39)-C(40) & $1.41(2)$ & $\mathrm{C}(58)-\mathrm{C}(59)$ & $1.50(2)$ \\
\hline C(39)-H(119) & 0.9300 & $C(59)-C(68)$ & $1.43(2)$ \\
\hline C(40)-H(120) & 0.9300 & $\mathrm{C}(59)-\mathrm{C}(60)$ & $1.44(2)$ \\
\hline $\mathrm{C}(41)-\mathrm{H}(41)$ & 0.9600 & $\mathrm{C}(60)-\mathrm{C}(61)$ & $1.41(2)$ \\
\hline $\mathrm{C}(41)-\mathrm{H}(41 \mathrm{~A})$ & 0.9600 & $C(60)-C(65)$ & $1.42(3)$ \\
\hline $\mathrm{C}(41)-\mathrm{H}(41 \mathrm{~B})$ & 0.9600 & $\mathrm{C}(61)-\mathrm{C}(62)$ & $1.35(2)$ \\
\hline$C(42)-C(43)$ & $1.39(2)$ & $\mathrm{C}(61)-\mathrm{H}(131)$ & 0.9300 \\
\hline C(42)-C(47) & $1.40(2)$ & $\mathrm{C}(62)-\mathrm{C}(63)$ & $1.43(3)$ \\
\hline $\mathrm{C}(43)-\mathrm{C}(44)$ & $1.40(2)$ & $\mathrm{C}(62)-\mathrm{H}(132)$ & 0.9300 \\
\hline C(43)-H(170) & 0.9300 & $C(63)-C(64)$ & $1.31(3)$ \\
\hline C(44)-C(45) & $1.39(3)$ & $\mathrm{C}(63)-\mathrm{H}(133)$ & 0.9300 \\
\hline $\mathrm{C}(44)-\mathrm{H}(122)$ & 0.9300 & $C(64)-C(65)$ & $1.43(2)$ \\
\hline $\mathrm{C}(45)-\mathrm{C}(121)$ & $1.37(3)$ & $\mathrm{C}(64)-\mathrm{H}(134)$ & 0.9300 \\
\hline $\mathrm{C}(45)-\mathrm{C}(48)$ & $1.49(3)$ & $C(65)-C(66)$ & $1.38(2)$ \\
\hline $\mathrm{C}(47)-\mathrm{C}(121)$ & $1.43(3)$ & $C(66)-C(67)$ & $1.37(2)$ \\
\hline $\mathrm{C}(47)-\mathrm{H}(179)$ & 0.9300 & $\mathrm{C}(66)-\mathrm{H}(135)$ & 0.9300 \\
\hline $\mathrm{C}(48)-\mathrm{H}(48)$ & 0.9600 & $\mathrm{C}(67)-\mathrm{C}(68)$ & $1.40(2)$ \\
\hline $\mathrm{C}(48)-\mathrm{H}(48 \mathrm{~A})$ & 0.9600 & $\mathrm{C}(67)-\mathrm{H}(136)$ & 0.9300 \\
\hline $\mathrm{C}(48)-\mathrm{H}(48 \mathrm{~B})$ & 0.9600 & $C(69)-C(70)$ & $1.34(3)$ \\
\hline
\end{tabular}




\begin{tabular}{|c|c|c|c|}
\hline$C(69)-C(74)$ & $1.40(2)$ & $\mathrm{C}(87)-\mathrm{H}(182)$ & 0.9300 \\
\hline$C(70)-C(71)$ & $1.40(3)$ & $\mathrm{C}(88)-\mathrm{H}(148)$ & 0.9300 \\
\hline $\mathrm{C}(70)-\mathrm{H}(137)$ & 0.9300 & C(89)-H(89) & 0.9600 \\
\hline $\mathrm{C}(71)-\mathrm{C}(72)$ & $1.40(3)$ & $\mathrm{C}(89)-\mathrm{H}(89 \mathrm{~A})$ & 0.9600 \\
\hline $\mathrm{C}(71)-\mathrm{H}(138)$ & 0.9300 & $\mathrm{C}(89)-\mathrm{H}(89 \mathrm{~B})$ & 0.9600 \\
\hline $\mathrm{C}(72)-\mathrm{C}(73)$ & $1.36(3)$ & $\mathrm{C}(90)-\mathrm{C}(95)$ & $1.35(2)$ \\
\hline$C(72)-C(75)$ & $1.51(3)$ & $\mathrm{C}(90)-\mathrm{C}(91)$ & $1.40(2)$ \\
\hline$C(73)-C(74)$ & $1.38(3)$ & $\mathrm{C}(91)-\mathrm{C}(92)$ & $1.37(2)$ \\
\hline C(73)-H(139) & 0.9300 & $\mathrm{C}(91)-\mathrm{H}(149)$ & 0.9300 \\
\hline C(74)-H(140) & 0.9300 & $\mathrm{C}(92)-\mathrm{C}(93)$ & $1.39(3)$ \\
\hline $\mathrm{C}(75)-\mathrm{H}(75)$ & 0.9600 & $\mathrm{C}(92)-\mathrm{H}(150)$ & 0.9300 \\
\hline $\mathrm{C}(75)-\mathrm{H}(75 \mathrm{~A})$ & 0.9600 & C(93)-C(94) & $1.40(3)$ \\
\hline $\mathrm{C}(75)-\mathrm{H}(75 \mathrm{~B})$ & 0.9600 & $\mathrm{C}(93)-\mathrm{C}(96)$ & $1.49(3)$ \\
\hline C(76)-C(77) & $1.37(2)$ & C(94)-C(95) & $1.37(3)$ \\
\hline$C(76)-C(81)$ & $1.43(2)$ & $\mathrm{C}(94)-\mathrm{H}(151)$ & 0.9300 \\
\hline $\mathrm{C}(77)-\mathrm{C}(78)$ & $1.42(3)$ & $\mathrm{C}(95)-\mathrm{H}(152)$ & 0.9300 \\
\hline $\mathrm{C}(77)-\mathrm{H}(141)$ & 0.9300 & $\mathrm{C}(96)-\mathrm{H}(96)$ & 0.9600 \\
\hline C(78)-C(79) & $1.42(3)$ & $\mathrm{C}(96)-\mathrm{H}(96 \mathrm{~A})$ & 0.9600 \\
\hline $\mathrm{C}(78)-\mathrm{H}(142)$ & 0.9300 & $\mathrm{C}(96)-\mathrm{H}(96 \mathrm{~B})$ & 0.9600 \\
\hline C(79)-C(80) & $1.38(3)$ & $\mathrm{Sb}(1)-\mathrm{F}(4)$ & $1.75(3)$ \\
\hline $\mathrm{C}(79)-\mathrm{C}(82)$ & $1.48(3)$ & $\mathrm{Sb}(1)-\mathrm{F}(5)$ & $1.77(3)$ \\
\hline $\mathrm{C}(80)-\mathrm{C}(81)$ & $1.36(3)$ & $\mathrm{Sb}(1)-\mathrm{F}(2)$ & $1.82(2)$ \\
\hline $\mathrm{C}(80)-\mathrm{H}(143)$ & 0.9300 & $\mathrm{Sb}(1)-\mathrm{F}(1)$ & $1.83(2)$ \\
\hline $\mathrm{C}(81)-\mathrm{H}(144)$ & 0.9300 & $\mathrm{Sb}(1)-\mathrm{F}(3)$ & $1.83(3)$ \\
\hline C(82)-H(82) & 0.9600 & $\mathrm{Sb}(1)-\mathrm{F}(6)$ & $1.87(3)$ \\
\hline $\mathrm{C}(82)-\mathrm{H}(82 \mathrm{~A})$ & 0.9600 & $\mathrm{Cl}(13)-\mathrm{C}(109)$ & $1.74(5)$ \\
\hline $\mathrm{C}(82)-\mathrm{H}(82 \mathrm{~B})$ & 0.9600 & $\mathrm{Cl}(13)-\mathrm{C}(103)$ & $1.79(5)$ \\
\hline C(83)-C(84) & $1.36(2)$ & $\mathrm{Cl}(14)-\mathrm{C}(109)$ & $1.83(5)$ \\
\hline $\mathrm{C}(83)-\mathrm{C}(88)$ & $1.43(2)$ & $\mathrm{Cl}(14)-\mathrm{C}(103)$ & $1.91(5)$ \\
\hline C(84)-C(85) & $1.41(2)$ & $C(103)-C(109)$ & $1.31(6)$ \\
\hline C(84)-H(145) & 0.9300 & $\mathrm{Cl}(9)-\mathrm{C}(120)$ & $1.80(6)$ \\
\hline $\mathrm{C}(85)-\mathrm{C}(86)$ & $1.42(2)$ & $\mathrm{Cl}(10)-\mathrm{C}(120)$ & $1.76(6)$ \\
\hline $\mathrm{C}(85)-\mathrm{H}(146)$ & 0.9300 & $\mathrm{Cl}(11)-\mathrm{C}(119)$ & $1.38(2)$ \\
\hline C(86)-C(87) & $1.37(3)$ & $\mathrm{Cl}(12)-\mathrm{C}(119)$ & $1.64(2)$ \\
\hline C(86)-C(89) & $1.50(2)$ & $\mathrm{C}(106)-\mathrm{H}(183)$ & 0.9600 \\
\hline C(87)-C(88) & $1.36(3)$ & $\mathrm{C}(106)-\mathrm{H}(184)$ & 0.9600 \\
\hline
\end{tabular}




\begin{tabular}{|c|c|}
\hline C(106)-H(185) & 0.9600 \\
\hline C(119)-H(177) & 0.9700 \\
\hline C(119)-H(178) & 0.9700 \\
\hline $\mathrm{C}(121)-\mathrm{H}(180)$ & 0.9300 \\
\hline$P(2)-\operatorname{Ir}(1)-P(1)$ & $91.41(13)$ \\
\hline$P(2)-\operatorname{Ir}(1)-\operatorname{Ir}(2)$ & $128.57(10)$ \\
\hline$P(1)-\operatorname{Ir}(1)-\operatorname{Ir}(2)$ & $126.05(9)$ \\
\hline$P(3)-\operatorname{Ir}(2)-P(4)$ & $92.28(14)$ \\
\hline$P(3)-\operatorname{Ir}(2)-\operatorname{Ir}(1)$ & $130.84(10)$ \\
\hline$P(4)-\operatorname{Ir}(2)-\operatorname{Ir}(1)$ & $125.68(11)$ \\
\hline $\mathrm{C}(1)-\mathrm{P}(1)-\mathrm{C}(21)$ & $107.3(6)$ \\
\hline C(1)-P(1)-C(28) & $105.1(7)$ \\
\hline $\mathrm{C}(21)-\mathrm{P}(1)-\mathrm{C}(28)$ & $100.4(7)$ \\
\hline C(1)-P(1)-Ir(1) & $105.5(5)$ \\
\hline $\mathrm{C}(21)-\mathrm{P}(1)-\operatorname{Ir}(1)$ & $122.1(5)$ \\
\hline $\mathrm{C}(28)-\mathrm{P}(1)-\operatorname{Ir}(1)$ & $115.1(5)$ \\
\hline $\mathrm{C}(35)-\mathrm{P}(2)-\mathrm{C}(20)$ & $102.4(7)$ \\
\hline $\mathrm{C}(35)-\mathrm{P}(2)-\mathrm{C}(42)$ & $105.3(7)$ \\
\hline $\mathrm{C}(20)-\mathrm{P}(2)-\mathrm{C}(42)$ & $104.3(7)$ \\
\hline $\mathrm{C}(35)-\mathrm{P}(2)-\operatorname{Ir}(1)$ & $117.3(6)$ \\
\hline$C(20)-P(2)-\operatorname{Ir}(1)$ & $116.2(5)$ \\
\hline $\mathrm{C}(42)-\mathrm{P}(2)-\operatorname{Ir}(1)$ & $110.0(5)$ \\
\hline $\mathrm{C}(76)-\mathrm{P}(3)-\mathrm{C}(69)$ & $105.1(7)$ \\
\hline $\mathrm{C}(76)-\mathrm{P}(3)-\mathrm{C}(49)$ & $106.1(7)$ \\
\hline $\mathrm{C}(69)-\mathrm{P}(3)-\mathrm{C}(49)$ & $104.2(8)$ \\
\hline$C(76)-P(3)-\operatorname{Ir}(2)$ & $116.3(5)$ \\
\hline $\mathrm{C}(69)-\mathrm{P}(3)-\operatorname{Ir}(2)$ & $110.7(6)$ \\
\hline C(49)-P(3)- $\operatorname{Ir}(2)$ & $113.3(5)$ \\
\hline $\mathrm{C}(90)-\mathrm{P}(4)-\mathrm{C}(83)$ & $104.4(7)$ \\
\hline $\mathrm{C}(90)-\mathrm{P}(4)-\mathrm{C}(68)$ & $105.5(7)$ \\
\hline $\mathrm{C}(83)-\mathrm{P}(4)-\mathrm{C}(68)$ & $102.5(7)$ \\
\hline C(90)-P(4)-Ir(2) & $118.5(6)$ \\
\hline C(83)-P(4)-Ir(2) & $110.4(5)$ \\
\hline$C(68)-P(4)-\operatorname{Ir}(2)$ & $113.9(5)$ \\
\hline$C(10)-C(1)-C(2)$ & $118.8(13)$ \\
\hline
\end{tabular}

\begin{tabular}{|c|c|}
\hline$C(10)-C(1)-P(1)$ & $122.1(11)$ \\
\hline$C(2)-C(1)-P(1)$ & $117.8(10)$ \\
\hline$C(3)-C(2)-C(1)$ & 121.2(13) \\
\hline $\mathrm{C}(3)-\mathrm{C}(2)-\mathrm{H}(97)$ & 119.4 \\
\hline $\mathrm{C}(1)-\mathrm{C}(2)-\mathrm{H}(97)$ & 119.4 \\
\hline$C(2)-C(3)-C(4)$ & $120.6(14)$ \\
\hline $\mathrm{C}(2)-\mathrm{C}(3)-\mathrm{H}(98)$ & 119.7 \\
\hline $\mathrm{C}(4)-\mathrm{C}(3)-\mathrm{H}(98)$ & 119.7 \\
\hline$C(9)-C(4)-C(3)$ & $119.9(13)$ \\
\hline$C(9)-C(4)-C(5)$ & $120.4(15)$ \\
\hline$C(3)-C(4)-C(5)$ & $119.6(14)$ \\
\hline$C(6)-C(5)-C(4)$ & $117.8(16)$ \\
\hline $\mathrm{C}(6)-\mathrm{C}(5)-\mathrm{H}(99)$ & 121.1 \\
\hline $\mathrm{C}(4)-\mathrm{C}(5)-\mathrm{H}(99)$ & 121.1 \\
\hline$C(5)-C(6)-C(7)$ & $121.8(15)$ \\
\hline $\mathrm{C}(5)-\mathrm{C}(6)-\mathrm{H}(100)$ & 119.1 \\
\hline $\mathrm{C}(7)-\mathrm{C}(6)-\mathrm{H}(100)$ & 119.1 \\
\hline$C(8)-C(7)-C(6)$ & $122.2(17)$ \\
\hline $\mathrm{C}(8)-\mathrm{C}(7)-\mathrm{H}(101)$ & 118.9 \\
\hline $\mathrm{C}(6)-\mathrm{C}(7)-\mathrm{H}(101)$ & 118.9 \\
\hline $\mathrm{C}(7)-\mathrm{C}(8)-\mathrm{C}(9)$ & $119.0(16)$ \\
\hline $\mathrm{C}(7)-\mathrm{C}(8)-\mathrm{H}(102)$ & 120.5 \\
\hline $\mathrm{C}(9)-\mathrm{C}(8)-\mathrm{H}(102)$ & 120.5 \\
\hline$C(4)-C(9)-C(10)$ & 119.7(13) \\
\hline $\mathrm{C}(4)-\mathrm{C}(9)-\mathrm{C}(8)$ & $118.7(13)$ \\
\hline $\mathrm{C}(10)-\mathrm{C}(9)-\mathrm{C}(8)$ & $121.5(13)$ \\
\hline$C(1)-C(10)-C(9)$ & $119.4(13)$ \\
\hline$C(1)-C(10)-C(11)$ & $120.7(13)$ \\
\hline$C(9)-C(10)-C(11)$ & $119.9(12)$ \\
\hline$C(20)-C(11)-C(12)$ & $121.2(13)$ \\
\hline$C(20)-C(11)-C(10)$ & $119.3(12)$ \\
\hline$C(12)-C(11)-C(10)$ & $119.4(13)$ \\
\hline$C(11)-C(12)-C(17)$ & $118.4(14)$ \\
\hline$C(11)-C(12)-C(13)$ & $123.1(15)$ \\
\hline$C(17)-C(12)-C(13)$ & $118.3(14)$ \\
\hline$C(14)-C(13)-C(12)$ & $119.7(16)$ \\
\hline
\end{tabular}




\begin{tabular}{|c|c|c|c|}
\hline C(14)-C(13)-H(103) & 120.2 & $\mathrm{C}(26)-\mathrm{C}(25)-\mathrm{H}(181)$ & 119.2 \\
\hline $\mathrm{C}(12)-\mathrm{C}(13)-\mathrm{H}(103)$ & 120.2 & $\mathrm{C}(24)-\mathrm{C}(25)-\mathrm{H}(181)$ & 119.2 \\
\hline $\mathrm{C}(13)-\mathrm{C}(14)-\mathrm{C}(15)$ & $122.8(18)$ & $C(25)-C(26)-C(21)$ & $119.7(14)$ \\
\hline $\mathrm{C}(13)-\mathrm{C}(14)-\mathrm{H}(104)$ & 118.6 & $C(25)-C(26)-H(112)$ & 120.1 \\
\hline $\mathrm{C}(15)-\mathrm{C}(14)-\mathrm{H}(104)$ & 118.6 & $\mathrm{C}(21)-\mathrm{C}(26)-\mathrm{H}(112)$ & 120.1 \\
\hline $\mathrm{C}(14)-\mathrm{C}(15)-\mathrm{C}(16)$ & $118.5(16)$ & $\mathrm{C}(24)-\mathrm{C}(27)-\mathrm{H}(27)$ & 109.5 \\
\hline $\mathrm{C}(14)-\mathrm{C}(15)-\mathrm{H}(105)$ & 120.8 & $\mathrm{C}(24)-\mathrm{C}(27)-\mathrm{H}(27 \mathrm{~A})$ & 109.5 \\
\hline $\mathrm{C}(16)-\mathrm{C}(15)-\mathrm{H}(105)$ & 120.8 & $\mathrm{H}(27)-\mathrm{C}(27)-\mathrm{H}(27 \mathrm{~A})$ & 109.5 \\
\hline$C(17)-C(16)-C(15)$ & $120.6(17)$ & $\mathrm{C}(24)-\mathrm{C}(27)-\mathrm{H}(27 \mathrm{~B})$ & 109.5 \\
\hline $\mathrm{C}(17)-\mathrm{C}(16)-\mathrm{H}(106)$ & 119.7 & $\mathrm{H}(27)-\mathrm{C}(27)-\mathrm{H}(27 \mathrm{~B})$ & 109.5 \\
\hline $\mathrm{C}(15)-\mathrm{C}(16)-\mathrm{H}(106)$ & 119.7 & $\mathrm{H}(27 \mathrm{~A})-\mathrm{C}(27)-\mathrm{H}(27 \mathrm{~B})$ & 109.5 \\
\hline$C(16)-C(17)-C(18)$ & $120.1(15)$ & $\mathrm{C}(33)-\mathrm{C}(28)-\mathrm{C}(29)$ & $118.2(14)$ \\
\hline $\mathrm{C}(16)-\mathrm{C}(17)-\mathrm{C}(12)$ & 119.7(16) & $\mathrm{C}(33)-\mathrm{C}(28)-\mathrm{P}(1)$ & $122.8(11)$ \\
\hline $\mathrm{C}(18)-\mathrm{C}(17)-\mathrm{C}(12)$ & $120.0(14)$ & $\mathrm{C}(29)-\mathrm{C}(28)-\mathrm{P}(1)$ & $119.0(12)$ \\
\hline $\mathrm{C}(19)-\mathrm{C}(18)-\mathrm{C}(17)$ & $118.9(15)$ & $\mathrm{C}(30)-\mathrm{C}(29)-\mathrm{C}(28)$ & $119.1(15)$ \\
\hline C(19)-C(18)-H(107) & 120.6 & $\mathrm{C}(30)-\mathrm{C}(29)-\mathrm{H}(113)$ & 120.4 \\
\hline $\mathrm{C}(17)-\mathrm{C}(18)-\mathrm{H}(107)$ & 120.6 & $\mathrm{C}(28)-\mathrm{C}(29)-\mathrm{H}(113)$ & 120.4 \\
\hline $\mathrm{C}(18)-\mathrm{C}(19)-\mathrm{C}(20)$ & $123.4(15)$ & $\mathrm{C}(29)-\mathrm{C}(30)-\mathrm{C}(31)$ & $123.5(14)$ \\
\hline C(18)-C(19)-H(108) & 118.3 & $\mathrm{C}(29)-\mathrm{C}(30)-\mathrm{H}(114)$ & 118.2 \\
\hline $\mathrm{C}(20)-\mathrm{C}(19)-\mathrm{H}(108)$ & 118.3 & $\mathrm{C}(31)-\mathrm{C}(30)-\mathrm{H}(114)$ & 118.2 \\
\hline $\mathrm{C}(11)-\mathrm{C}(20)-\mathrm{C}(19)$ & $117.9(13)$ & $\mathrm{C}(30)-\mathrm{C}(31)-\mathrm{C}(32)$ & $116.4(15)$ \\
\hline $\mathrm{C}(11)-\mathrm{C}(20)-\mathrm{P}(2)$ & $122.0(11)$ & $\mathrm{C}(30)-\mathrm{C}(31)-\mathrm{C}(106)$ & $120.9(15)$ \\
\hline C(19)-C(20)-P(2) & $119.6(11)$ & $\mathrm{C}(32)-\mathrm{C}(31)-\mathrm{C}(106)$ & $122.5(16)$ \\
\hline $\mathrm{C}(22)-\mathrm{C}(21)-\mathrm{C}(26)$ & $118.5(13)$ & $\mathrm{C}(33)-\mathrm{C}(32)-\mathrm{C}(31)$ & $121.4(16)$ \\
\hline $\mathrm{C}(22)-\mathrm{C}(21)-\mathrm{P}(1)$ & $121.4(12)$ & $\mathrm{C}(33)-\mathrm{C}(32)-\mathrm{H}(115)$ & 119.3 \\
\hline $\mathrm{C}(26)-\mathrm{C}(21)-\mathrm{P}(1)$ & $120.0(11)$ & $\mathrm{C}(31)-\mathrm{C}(32)-\mathrm{H}(115)$ & 119.3 \\
\hline $\mathrm{C}(23)-\mathrm{C}(22)-\mathrm{C}(21)$ & $120.1(15)$ & $\mathrm{C}(32)-\mathrm{C}(33)-\mathrm{C}(28)$ & $121.4(14)$ \\
\hline $\mathrm{C}(23)-\mathrm{C}(22)-\mathrm{H}(109)$ & 120.0 & $\mathrm{C}(32)-\mathrm{C}(33)-\mathrm{H}(116)$ & 119.3 \\
\hline $\mathrm{C}(21)-\mathrm{C}(22)-\mathrm{H}(109)$ & 120.0 & $\mathrm{C}(28)-\mathrm{C}(33)-\mathrm{H}(116)$ & 119.3 \\
\hline $\mathrm{C}(24)-\mathrm{C}(23)-\mathrm{C}(22)$ & $120.7(16)$ & $C(36)-C(35)-C(40)$ & $118.1(14)$ \\
\hline $\mathrm{C}(24)-\mathrm{C}(23)-\mathrm{H}(110)$ & 119.7 & $\mathrm{C}(36)-\mathrm{C}(35)-\mathrm{P}(2)$ & $122.0(13)$ \\
\hline $\mathrm{C}(22)-\mathrm{C}(23)-\mathrm{H}(110)$ & 119.7 & $\mathrm{C}(40)-\mathrm{C}(35)-\mathrm{P}(2)$ & $119.9(11)$ \\
\hline $\mathrm{C}(23)-\mathrm{C}(24)-\mathrm{C}(25)$ & $119.3(14)$ & $\mathrm{C}(35)-\mathrm{C}(36)-\mathrm{C}(37)$ & $120.8(16)$ \\
\hline $\mathrm{C}(23)-\mathrm{C}(24)-\mathrm{C}(27)$ & $120.9(16)$ & $\mathrm{C}(35)-\mathrm{C}(36)-\mathrm{H}(117)$ & 119.6 \\
\hline $\mathrm{C}(25)-\mathrm{C}(24)-\mathrm{C}(27)$ & $119.7(16)$ & $\mathrm{C}(37)-\mathrm{C}(36)-\mathrm{H}(117)$ & 119.6 \\
\hline$C(26)-C(25)-C(24)$ & $121.5(15)$ & $\mathrm{C}(38)-\mathrm{C}(37)-\mathrm{C}(36)$ & $120.6(15)$ \\
\hline
\end{tabular}




\begin{tabular}{|c|c|c|c|}
\hline $\mathrm{C}(38)-\mathrm{C}(37)-\mathrm{H}(118)$ & 119.7 & $\mathrm{H}(48)-\mathrm{C}(48)-\mathrm{H}(48 \mathrm{~B})$ & 109.5 \\
\hline $\mathrm{C}(36)-\mathrm{C}(37)-\mathrm{H}(118)$ & 119.7 & $\mathrm{H}(48 \mathrm{~A})-\mathrm{C}(48)-\mathrm{H}(48 \mathrm{~B})$ & 109.5 \\
\hline C(39)-C(38)-C(37) & $120.2(15)$ & $\mathrm{C}(58)-\mathrm{C}(49)-\mathrm{C}(50)$ & $119.6(16)$ \\
\hline C(39)-C(38)-C(41) & $119.3(17)$ & $\mathrm{C}(58)-\mathrm{C}(49)-\mathrm{P}(3)$ & $121.2(14)$ \\
\hline C(37)-C(38)-C(41) & $120.4(15)$ & $\mathrm{C}(50)-\mathrm{C}(49)-\mathrm{P}(3)$ & $119.0(13)$ \\
\hline C(38)-C(39)-C(40) & $120.8(17)$ & $\mathrm{C}(51)-\mathrm{C}(50)-\mathrm{C}(49)$ & $120.7(19)$ \\
\hline C(38)-C(39)-H(119) & 119.6 & $\mathrm{C}(51)-\mathrm{C}(50)-\mathrm{H}(125)$ & 119.6 \\
\hline C(40)-C(39)-H(119) & 119.6 & $\mathrm{C}(49)-\mathrm{C}(50)-\mathrm{H}(125)$ & 119.6 \\
\hline C(39)-C(40)-C(35) & $119.5(14)$ & $\mathrm{C}(50)-\mathrm{C}(51)-\mathrm{C}(52)$ & $121.7(19)$ \\
\hline C(39)-C(40)-H(120) & 120.3 & $\mathrm{C}(50)-\mathrm{C}(51)-\mathrm{H}(126)$ & 119.2 \\
\hline $\mathrm{C}(35)-\mathrm{C}(40)-\mathrm{H}(120)$ & 120.3 & $\mathrm{C}(52)-\mathrm{C}(51)-\mathrm{H}(126)$ & 119.2 \\
\hline $\mathrm{C}(38)-\mathrm{C}(41)-\mathrm{H}(41)$ & 109.5 & C(51)-C(52)-C(57) & $119.3(15)$ \\
\hline $\mathrm{C}(38)-\mathrm{C}(41)-\mathrm{H}(41 \mathrm{~A})$ & 109.5 & $\mathrm{C}(51)-\mathrm{C}(52)-\mathrm{C}(53)$ & $121.5(18)$ \\
\hline $\mathrm{H}(41)-\mathrm{C}(41)-\mathrm{H}(41 \mathrm{~A})$ & 109.5 & $\mathrm{C}(57)-\mathrm{C}(52)-\mathrm{C}(53)$ & $119.2(17)$ \\
\hline C(38)-C(41)-H(41B) & 109.5 & $\mathrm{C}(54)-\mathrm{C}(53)-\mathrm{C}(52)$ & $118.5(19)$ \\
\hline $\mathrm{H}(41)-\mathrm{C}(41)-\mathrm{H}(41 \mathrm{~B})$ & 109.5 & $\mathrm{C}(54)-\mathrm{C}(53)-\mathrm{H}(127)$ & 120.7 \\
\hline $\mathrm{H}(41 \mathrm{~A})-\mathrm{C}(41)-\mathrm{H}(41 \mathrm{~B})$ & 109.5 & $\mathrm{C}(52)-\mathrm{C}(53)-\mathrm{H}(127)$ & 120.7 \\
\hline$C(43)-C(42)-C(47)$ & $119.4(15)$ & $C(53)-C(54)-C(55)$ & $121.0(17)$ \\
\hline $\mathrm{C}(43)-\mathrm{C}(42)-\mathrm{P}(2)$ & $119.3(12)$ & $\mathrm{C}(53)-\mathrm{C}(54)-\mathrm{H}(128)$ & 119.5 \\
\hline $\mathrm{C}(47)-\mathrm{C}(42)-\mathrm{P}(2)$ & $121.1(12)$ & $\mathrm{C}(55)-\mathrm{C}(54)-\mathrm{H}(128)$ & 119.5 \\
\hline$C(42)-C(43)-C(44)$ & $120.4(16)$ & $\mathrm{C}(56)-\mathrm{C}(55)-\mathrm{C}(54)$ & $122(2)$ \\
\hline $\mathrm{C}(42)-\mathrm{C}(43)-\mathrm{H}(170)$ & 119.8 & $\mathrm{C}(56)-\mathrm{C}(55)-\mathrm{H}(129)$ & 118.9 \\
\hline $\mathrm{C}(44)-\mathrm{C}(43)-\mathrm{H}(170)$ & 119.8 & $\mathrm{C}(54)-\mathrm{C}(55)-\mathrm{H}(129)$ & 118.9 \\
\hline$C(45)-C(44)-C(43)$ & $121.3(16)$ & $\mathrm{C}(55)-\mathrm{C}(56)-\mathrm{C}(57)$ & $119.9(19)$ \\
\hline $\mathrm{C}(45)-\mathrm{C}(44)-\mathrm{H}(122)$ & 119.4 & $\mathrm{C}(55)-\mathrm{C}(56)-\mathrm{H}(130)$ & 120.0 \\
\hline $\mathrm{C}(43)-\mathrm{C}(44)-\mathrm{H}(122)$ & 119.4 & $\mathrm{C}(57)-\mathrm{C}(56)-\mathrm{H}(130)$ & 120.0 \\
\hline $\mathrm{C}(121)-\mathrm{C}(45)-\mathrm{C}(44)$ & $118.5(16)$ & $\mathrm{C}(52)-\mathrm{C}(57)-\mathrm{C}(58)$ & $118.4(15)$ \\
\hline $\mathrm{C}(121)-\mathrm{C}(45)-\mathrm{C}(48)$ & $119.6(18)$ & $\mathrm{C}(52)-\mathrm{C}(57)-\mathrm{C}(56)$ & $119.0(15)$ \\
\hline $\mathrm{C}(44)-\mathrm{C}(45)-\mathrm{C}(48)$ & $121.9(17)$ & $\mathrm{C}(58)-\mathrm{C}(57)-\mathrm{C}(56)$ & $122.6(16)$ \\
\hline $\mathrm{C}(42)-\mathrm{C}(47)-\mathrm{C}(121)$ & $118.9(16)$ & $\mathrm{C}(49)-\mathrm{C}(58)-\mathrm{C}(57)$ & $120.2(15)$ \\
\hline $\mathrm{C}(42)-\mathrm{C}(47)-\mathrm{H}(179)$ & 120.5 & $\mathrm{C}(49)-\mathrm{C}(58)-\mathrm{C}(59)$ & $122.5(14)$ \\
\hline $\mathrm{C}(121)-\mathrm{C}(47)-\mathrm{H}(179)$ & 120.5 & $\mathrm{C}(57)-\mathrm{C}(58)-\mathrm{C}(59)$ & $117.3(13)$ \\
\hline $\mathrm{C}(45)-\mathrm{C}(48)-\mathrm{H}(48)$ & 109.5 & $\mathrm{C}(68)-\mathrm{C}(59)-\mathrm{C}(60)$ & $118.7(14)$ \\
\hline $\mathrm{C}(45)-\mathrm{C}(48)-\mathrm{H}(48 \mathrm{~A})$ & 109.5 & $\mathrm{C}(68)-\mathrm{C}(59)-\mathrm{C}(58)$ & $123.0(13)$ \\
\hline $\mathrm{H}(48)-\mathrm{C}(48)-\mathrm{H}(48 \mathrm{~A})$ & 109.5 & $\mathrm{C}(60)-\mathrm{C}(59)-\mathrm{C}(58)$ & $118.3(13)$ \\
\hline $\mathrm{C}(45)-\mathrm{C}(48)-\mathrm{H}(48 \mathrm{~B})$ & 109.5 & $\mathrm{C}(61)-\mathrm{C}(60)-\mathrm{C}(65)$ & $118.4(15)$ \\
\hline
\end{tabular}




\begin{tabular}{|c|c|}
\hline $\mathrm{C}(61)-\mathrm{C}(60)-\mathrm{C}(59)$ & $122.3(16)$ \\
\hline$C(65)-C(60)-C(59)$ & $119.1(15)$ \\
\hline$C(62)-C(61)-C(60)$ & $120.5(18)$ \\
\hline $\mathrm{C}(62)-\mathrm{C}(61)-\mathrm{H}(131)$ & 119.8 \\
\hline $\mathrm{C}(60)-\mathrm{C}(61)-\mathrm{H}(131)$ & 119.8 \\
\hline $\mathrm{C}(61)-\mathrm{C}(62)-\mathrm{C}(63)$ & $121.2(19)$ \\
\hline $\mathrm{C}(61)-\mathrm{C}(62)-\mathrm{H}(132)$ & 119.4 \\
\hline $\mathrm{C}(63)-\mathrm{C}(62)-\mathrm{H}(132)$ & 119.4 \\
\hline $\mathrm{C}(64)-\mathrm{C}(63)-\mathrm{C}(62)$ & $119(2)$ \\
\hline $\mathrm{C}(64)-\mathrm{C}(63)-\mathrm{H}(133)$ & 120.6 \\
\hline $\mathrm{C}(62)-\mathrm{C}(63)-\mathrm{H}(133)$ & 120.6 \\
\hline$C(63)-C(64)-C(65)$ & $123(2)$ \\
\hline $\mathrm{C}(63)-\mathrm{C}(64)-\mathrm{H}(134)$ & 118.7 \\
\hline $\mathrm{C}(65)-\mathrm{C}(64)-\mathrm{H}(134)$ & 118.7 \\
\hline$C(66)-C(65)-C(60)$ & $119.7(15)$ \\
\hline $\mathrm{C}(66)-\mathrm{C}(65)-\mathrm{C}(64)$ & $121.9(17)$ \\
\hline $\mathrm{C}(60)-\mathrm{C}(65)-\mathrm{C}(64)$ & $118.4(16)$ \\
\hline $\mathrm{C}(67)-\mathrm{C}(66)-\mathrm{C}(65)$ & $122.1(16)$ \\
\hline $\mathrm{C}(67)-\mathrm{C}(66)-\mathrm{H}(135)$ & 118.9 \\
\hline $\mathrm{C}(65)-\mathrm{C}(66)-\mathrm{H}(135)$ & 118.9 \\
\hline $\mathrm{C}(66)-\mathrm{C}(67)-\mathrm{C}(68)$ & $120.5(16)$ \\
\hline $\mathrm{C}(66)-\mathrm{C}(67)-\mathrm{H}(136)$ & 119.7 \\
\hline $\mathrm{C}(68)-\mathrm{C}(67)-\mathrm{H}(136)$ & 119.7 \\
\hline $\mathrm{C}(67)-\mathrm{C}(68)-\mathrm{C}(59)$ & $119.8(14)$ \\
\hline $\mathrm{C}(67)-\mathrm{C}(68)-\mathrm{P}(4)$ & $119.9(12)$ \\
\hline C(59)-C(68)-P(4) & $119.2(12)$ \\
\hline$C(70)-C(69)-C(74)$ & $118.0(17)$ \\
\hline $\mathrm{C}(70)-\mathrm{C}(69)-\mathrm{P}(3)$ & $121.3(13)$ \\
\hline $\mathrm{C}(74)-\mathrm{C}(69)-\mathrm{P}(3)$ & $120.7(15)$ \\
\hline $\mathrm{C}(69)-\mathrm{C}(70)-\mathrm{C}(71)$ & $121.5(19)$ \\
\hline $\mathrm{C}(69)-\mathrm{C}(70)-\mathrm{H}(137)$ & 119.3 \\
\hline $\mathrm{C}(71)-\mathrm{C}(70)-\mathrm{H}(137)$ & 119.3 \\
\hline $\mathrm{C}(70)-\mathrm{C}(71)-\mathrm{C}(72)$ & $120(2)$ \\
\hline $\mathrm{C}(70)-\mathrm{C}(71)-\mathrm{H}(138)$ & 119.8 \\
\hline $\mathrm{C}(72)-\mathrm{C}(71)-\mathrm{H}(138)$ & 119.8 \\
\hline$C(73)-C(72)-C(71)$ & $118(2)$ \\
\hline
\end{tabular}

\begin{tabular}{|c|c|}
\hline $\mathrm{C}(73)-\mathrm{C}(72)-\mathrm{C}(75)$ & $122(2)$ \\
\hline $\mathrm{C}(71)-\mathrm{C}(72)-\mathrm{C}(75)$ & $120(2)$ \\
\hline$C(72)-C(73)-C(74)$ & $120.9(18)$ \\
\hline $\mathrm{C}(72)-\mathrm{C}(73)-\mathrm{H}(139)$ & 119.5 \\
\hline $\mathrm{C}(74)-\mathrm{C}(73)-\mathrm{H}(139)$ & 119.5 \\
\hline C(73)-C(74)-C(69) & 121.3(19) \\
\hline $\mathrm{C}(73)-\mathrm{C}(74)-\mathrm{H}(140)$ & 119.3 \\
\hline $\mathrm{C}(69)-\mathrm{C}(74)-\mathrm{H}(140)$ & 119.3 \\
\hline $\mathrm{C}(72)-\mathrm{C}(75)-\mathrm{H}(75)$ & 109.5 \\
\hline $\mathrm{C}(72)-\mathrm{C}(75)-\mathrm{H}(75 \mathrm{~A})$ & 109.5 \\
\hline $\mathrm{H}(75)-\mathrm{C}(75)-\mathrm{H}(75 \mathrm{~A})$ & 109.5 \\
\hline $\mathrm{C}(72)-\mathrm{C}(75)-\mathrm{H}(75 \mathrm{~B})$ & 109.5 \\
\hline $\mathrm{H}(75)-\mathrm{C}(75)-\mathrm{H}(75 \mathrm{~B})$ & 109.5 \\
\hline $\mathrm{H}(75 \mathrm{~A})-\mathrm{C}(75)-\mathrm{H}(75 \mathrm{~B})$ & 109.5 \\
\hline$C(77)-C(76)-C(81)$ & $119.0(15)$ \\
\hline $\mathrm{C}(77)-\mathrm{C}(76)-\mathrm{P}(3)$ & $120.9(12)$ \\
\hline $\mathrm{C}(81)-\mathrm{C}(76)-\mathrm{P}(3)$ & $119.9(13)$ \\
\hline $\mathrm{C}(76)-\mathrm{C}(77)-\mathrm{C}(78)$ & $121.8(16)$ \\
\hline $\mathrm{C}(76)-\mathrm{C}(77)-\mathrm{H}(141)$ & 119.1 \\
\hline $\mathrm{C}(78)-\mathrm{C}(77)-\mathrm{H}(141)$ & 119.1 \\
\hline $\mathrm{C}(77)-\mathrm{C}(78)-\mathrm{C}(79)$ & $118.5(19)$ \\
\hline $\mathrm{C}(77)-\mathrm{C}(78)-\mathrm{H}(142)$ & 120.7 \\
\hline $\mathrm{C}(79)-\mathrm{C}(78)-\mathrm{H}(142)$ & 120.7 \\
\hline $\mathrm{C}(80)-\mathrm{C}(79)-\mathrm{C}(78)$ & $117.6(17)$ \\
\hline $\mathrm{C}(80)-\mathrm{C}(79)-\mathrm{C}(82)$ & $126(2)$ \\
\hline $\mathrm{C}(78)-\mathrm{C}(79)-\mathrm{C}(82)$ & $117(2)$ \\
\hline$C(81)-C(80)-C(79)$ & $124.7(16)$ \\
\hline C(81)-C(80)-H(143) & 117.7 \\
\hline $\mathrm{C}(79)-\mathrm{C}(80)-\mathrm{H}(143)$ & 117.7 \\
\hline $\mathrm{C}(80)-\mathrm{C}(81)-\mathrm{C}(76)$ & $118.3(18)$ \\
\hline C(80)-C(81)-H(144) & 120.8 \\
\hline $\mathrm{C}(76)-\mathrm{C}(81)-\mathrm{H}(144)$ & 120.8 \\
\hline $\mathrm{C}(79)-\mathrm{C}(82)-\mathrm{H}(82)$ & 109.5 \\
\hline $\mathrm{C}(79)-\mathrm{C}(82)-\mathrm{H}(82 \mathrm{~A})$ & 109.5 \\
\hline $\mathrm{H}(82)-\mathrm{C}(82)-\mathrm{H}(82 \mathrm{~A})$ & 109.5 \\
\hline $\mathrm{C}(79)-\mathrm{C}(82)-\mathrm{H}(82 \mathrm{~B})$ & 109.5 \\
\hline
\end{tabular}




\begin{tabular}{|c|c|c|c|}
\hline $\mathrm{H}(82)-\mathrm{C}(82)-\mathrm{H}(82 \mathrm{~B})$ & 109.5 & $\mathrm{C}(92)-\mathrm{C}(93)-\mathrm{C}(96)$ & $120.5(18)$ \\
\hline $\mathrm{H}(82 \mathrm{~A})-\mathrm{C}(82)-\mathrm{H}(82 \mathrm{~B})$ & 109.5 & $\mathrm{C}(94)-\mathrm{C}(93)-\mathrm{C}(96)$ & $123(2)$ \\
\hline C(84)-C(83)-C(88) & $118.4(15)$ & C(95)-C(94)-C(93) & $120(2)$ \\
\hline C(84)-C(83)-P(4) & $119.7(12)$ & $\mathrm{C}(95)-\mathrm{C}(94)-\mathrm{H}(151)$ & 120.1 \\
\hline $\mathrm{C}(88)-\mathrm{C}(83)-\mathrm{P}(4)$ & $121.9(13)$ & $\mathrm{C}(93)-\mathrm{C}(94)-\mathrm{H}(151)$ & 120.1 \\
\hline $\mathrm{C}(83)-\mathrm{C}(84)-\mathrm{C}(85)$ & $122.1(16)$ & $\mathrm{C}(90)-\mathrm{C}(95)-\mathrm{C}(94)$ & $123.4(18)$ \\
\hline C(83)-C(84)-H(145) & 119.0 & $\mathrm{C}(90)-\mathrm{C}(95)-\mathrm{H}(152)$ & 118.3 \\
\hline C(85)-C(84)-H(145) & 119.0 & $\mathrm{C}(94)-\mathrm{C}(95)-\mathrm{H}(152)$ & 118.3 \\
\hline $\mathrm{C}(84)-\mathrm{C}(85)-\mathrm{C}(86)$ & $119.1(18)$ & $\mathrm{C}(93)-\mathrm{C}(96)-\mathrm{H}(96)$ & 109.5 \\
\hline C(84)-C(85)-H(146) & 120.4 & $\mathrm{C}(93)-\mathrm{C}(96)-\mathrm{H}(96 \mathrm{~A})$ & 109.5 \\
\hline $\mathrm{C}(86)-\mathrm{C}(85)-\mathrm{H}(146)$ & 120.4 & $\mathrm{H}(96)-\mathrm{C}(96)-\mathrm{H}(96 \mathrm{~A})$ & 109.5 \\
\hline C(87)-C(86)-C(85) & $117.0(15)$ & $\mathrm{C}(93)-\mathrm{C}(96)-\mathrm{H}(96 \mathrm{~B})$ & 109.5 \\
\hline C(87)-C(86)-C(89) & $123.1(16)$ & $\mathrm{H}(96)-\mathrm{C}(96)-\mathrm{H}(96 \mathrm{~B})$ & 109.5 \\
\hline C(85)-C(86)-C(89) & $119.8(18)$ & $\mathrm{H}(96 \mathrm{~A})-\mathrm{C}(96)-\mathrm{H}(96 \mathrm{~B})$ & 109.5 \\
\hline C(88)-C(87)-C(86) & $124.2(15)$ & $\mathrm{F}(4)-\mathrm{Sb}(1)-\mathrm{F}(5)$ & $94.5(15)$ \\
\hline C(88)-C(87)-H(182) & 117.9 & $\mathrm{~F}(4)-\mathrm{Sb}(1)-\mathrm{F}(2)$ & $174.9(14)$ \\
\hline C(86)-C(87)-H(182) & 117.9 & $\mathrm{~F}(5)-\mathrm{Sb}(1)-\mathrm{F}(2)$ & $81.9(11)$ \\
\hline $\mathrm{C}(87)-\mathrm{C}(88)-\mathrm{C}(83)$ & $119.0(17)$ & $\mathrm{F}(4)-\mathrm{Sb}(1)-\mathrm{F}(1)$ & $90.8(13)$ \\
\hline C(87)-C(88)-H(148) & 120.5 & $\mathrm{~F}(5)-\mathrm{Sb}(1)-\mathrm{F}(1)$ & $92.7(11)$ \\
\hline $\mathrm{C}(83)-\mathrm{C}(88)-\mathrm{H}(148)$ & 120.5 & $\mathrm{~F}(2)-\mathrm{Sb}(1)-\mathrm{F}(1)$ & $85.8(10)$ \\
\hline C(86)-C(89)-H(89) & 109.5 & $\mathrm{~F}(4)-\mathrm{Sb}(1)-\mathrm{F}(3)$ & $93.8(14)$ \\
\hline C(86)-C(89)-H(89A) & 109.5 & $\mathrm{~F}(5)-\mathrm{Sb}(1)-\mathrm{F}(3)$ & $170.6(13)$ \\
\hline H(89)-C(89)-H(89A) & 109.5 & $\mathrm{~F}(2)-\mathrm{Sb}(1)-\mathrm{F}(3)$ & $90.1(12)$ \\
\hline C(86)-C(89)-H(89B) & 109.5 & $\mathrm{~F}(1)-\mathrm{Sb}(1)-\mathrm{F}(3)$ & $91.6(11)$ \\
\hline H(89)-C(89)-H(89B) & 109.5 & $\mathrm{~F}(4)-\mathrm{Sb}(1)-\mathrm{F}(6)$ & $87.9(14)$ \\
\hline H(89A)-C(89)-H(89B) & 109.5 & $\mathrm{~F}(5)-\mathrm{Sb}(1)-\mathrm{F}(6)$ & $90.5(12)$ \\
\hline C(95)-C(90)-C(91) & $117.8(16)$ & $\mathrm{F}(2)-\mathrm{Sb}(1)-\mathrm{F}(6)$ & $95.7(12)$ \\
\hline $\mathrm{C}(95)-\mathrm{C}(90)-\mathrm{P}(4)$ & $122.9(12)$ & $\mathrm{F}(1)-\mathrm{Sb}(1)-\mathrm{F}(6)$ & $176.7(11)$ \\
\hline C(91)-C(90)-P(4) & $119.3(14)$ & $\mathrm{F}(3)-\mathrm{Sb}(1)-\mathrm{F}(6)$ & $85.4(13)$ \\
\hline $\mathrm{C}(92)-\mathrm{C}(91)-\mathrm{C}(90)$ & $119.7(17)$ & $\mathrm{C}(109)-\mathrm{Cl}(13)-\mathrm{C}(103)$ & $43(2)$ \\
\hline C(92)-C(91)-H(149) & 120.2 & $\mathrm{C}(109)-\mathrm{Cl}(14)-\mathrm{C}(103)$ & $41(2)$ \\
\hline C(90)-C(91)-H(149) & 120.2 & $\mathrm{C}(109)-\mathrm{C}(103)-\mathrm{Cl}(13)$ & $66(3)$ \\
\hline C(91)-C(92)-C(93) & $122.3(16)$ & $\mathrm{C}(109)-\mathrm{C}(103)-\mathrm{Cl}(14)$ & $66(3)$ \\
\hline C(91)-C(92)-H(150) & 118.8 & $\mathrm{Cl}(13)-\mathrm{C}(103)-\mathrm{Cl}(14)$ & $104(2)$ \\
\hline C(93)-C(92)-H(150) & 118.8 & $\mathrm{C}(31)-\mathrm{C}(106)-\mathrm{H}(183)$ & 109.5 \\
\hline C(92)-C(93)-C(94) & $117.0(16)$ & $\mathrm{C}(31)-\mathrm{C}(106)-\mathrm{H}(184)$ & 109.5 \\
\hline
\end{tabular}




\begin{tabular}{lrlr}
$\mathrm{H}(183)-\mathrm{C}(106)-\mathrm{H}(184)$ & 109.5 & $\mathrm{Cl}(11)-\mathrm{C}(119)-\mathrm{H}(178)$ & 98.4 \\
$\mathrm{C}(31)-\mathrm{C}(106)-\mathrm{H}(185)$ & 109.5 & $\mathrm{Cl}(12)-\mathrm{C}(119)-\mathrm{H}(178)$ & 98.4 \\
$\mathrm{H}(183)-\mathrm{C}(106)-\mathrm{H}(185)$ & 109.5 & $\mathrm{H}(177)-\mathrm{C}(119)-\mathrm{H}(178)$ & 103.8 \\
$\mathrm{H}(184)-\mathrm{C}(106)-\mathrm{H}(185)$ & 109.5 & $\mathrm{Cl}(10)-\mathrm{C}(120)-\mathrm{Cl}(9)$ & $114(3)$ \\
$\mathrm{C}(103)-\mathrm{C}(109)-\mathrm{Cl}(13)$ & $70(3)$ & $\mathrm{C}(45)-\mathrm{C}(121)-\mathrm{C}(47)$ & $121.4(17)$ \\
$\mathrm{C}(103)-\mathrm{C}(109)-\mathrm{Cl}(14)$ & $73(3)$ & $\mathrm{C}(45)-\mathrm{C}(121)-\mathrm{H}(180)$ & 119.3 \\
$\mathrm{Cl}(13)-\mathrm{C}(109)-\mathrm{Cl}(14)$ & $110(3)$ & $\mathrm{C}(47)-\mathrm{C}(121)-\mathrm{H}(180)$ & 119.3 \\
$\mathrm{Cl}(11)-\mathrm{C}(119)-\mathrm{Cl}(12)$ & $152.5(17)$ & & \\
\cline { 2 - 2 } $\mathrm{Cl}(11)-\mathrm{C}(119)-\mathrm{H}(177)$ & 98.4 & Symmetry transformations used to \\
$\mathrm{Cl}(12)-\mathrm{C}(119)-\mathrm{H}(177)$ & 98.4 & generate equivalent atoms:
\end{tabular}




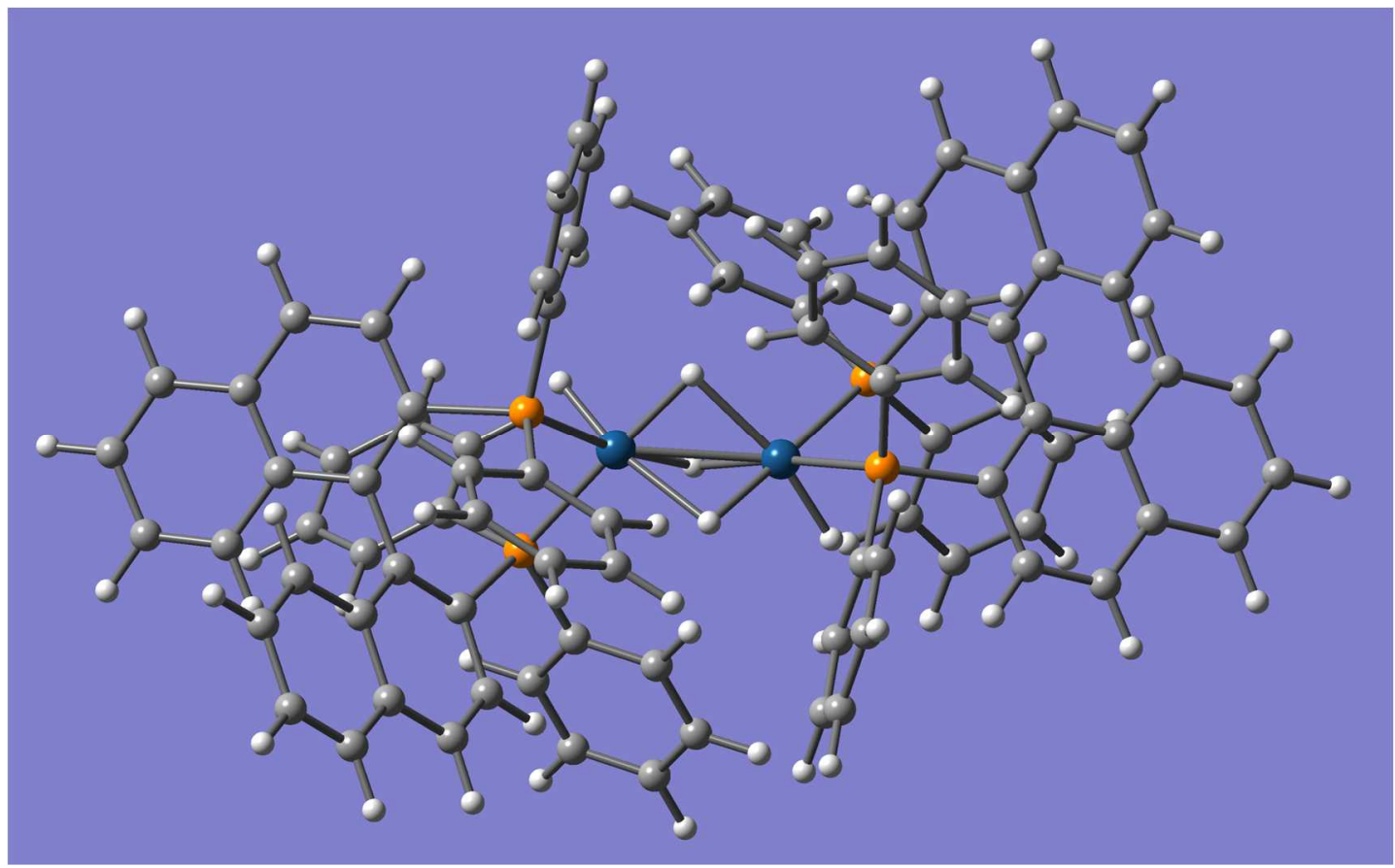

Figure S12. Optimized structure of 2a. 

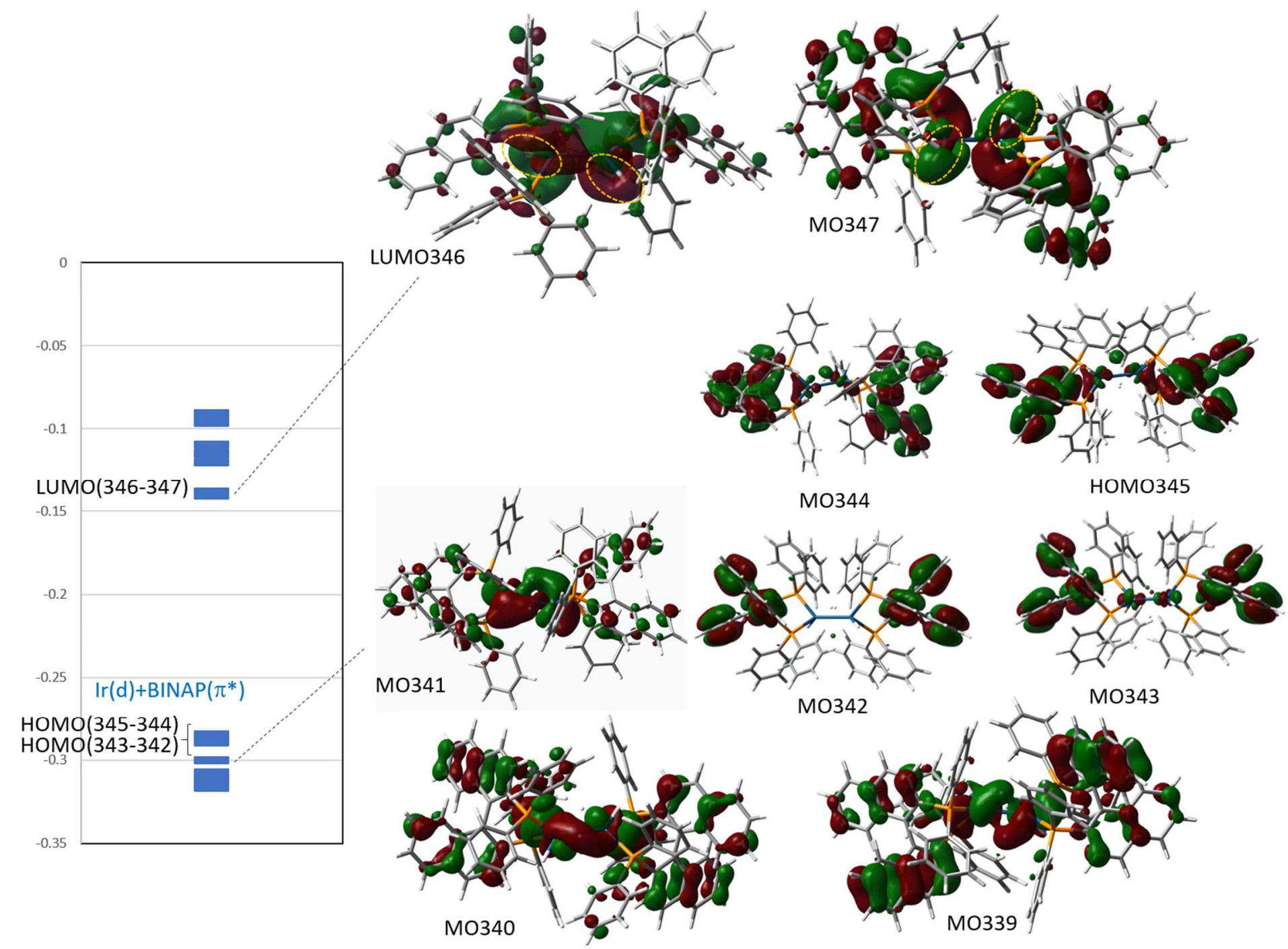

Figure S13. Frontier molecular orbitals of $\mathbf{2 a}$. 
(a)

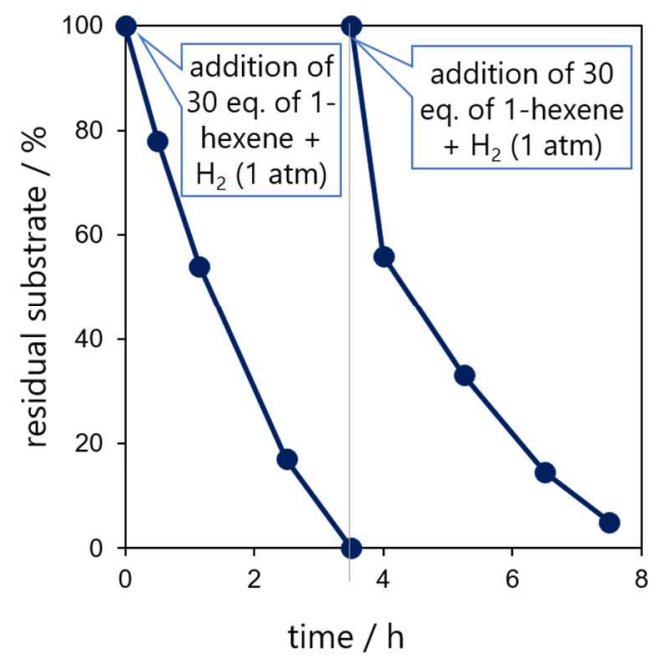

(b)

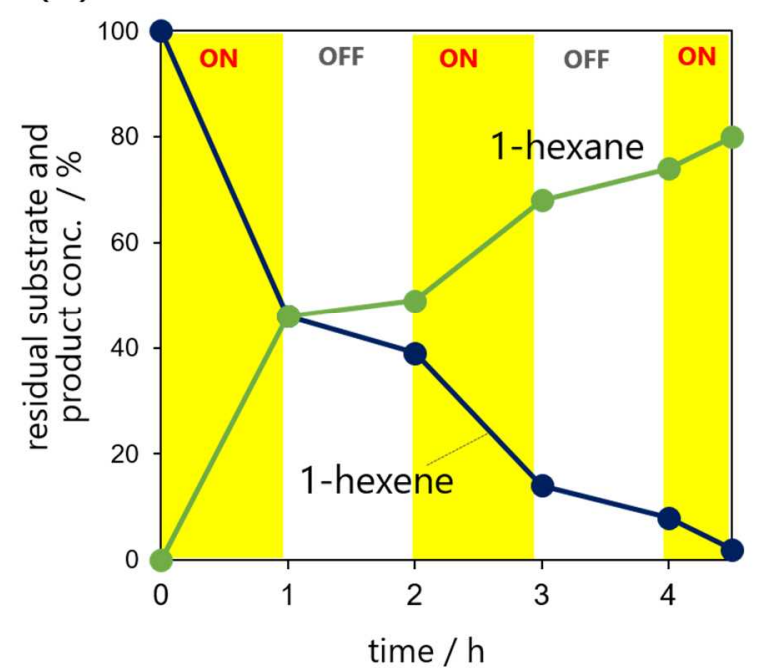

Figure S14. (a) Addition of 1-hexene (30 eq.) and $\mathrm{H}_{2}(1 \mathrm{~atm})$ after full conversion of 1-hexene after $1^{\text {st }}$ hydrogenation of 1-hexene under irradiation $(395 \mathrm{~nm}$ ) by catalyst 2a. (b) Time course for the catalytic hydrogenation of 1-hexene catalyzed by $\mathbf{2 a}$ under repeated on $(395 \mathrm{~nm}) / \mathrm{off}$ (dark) cycles. 

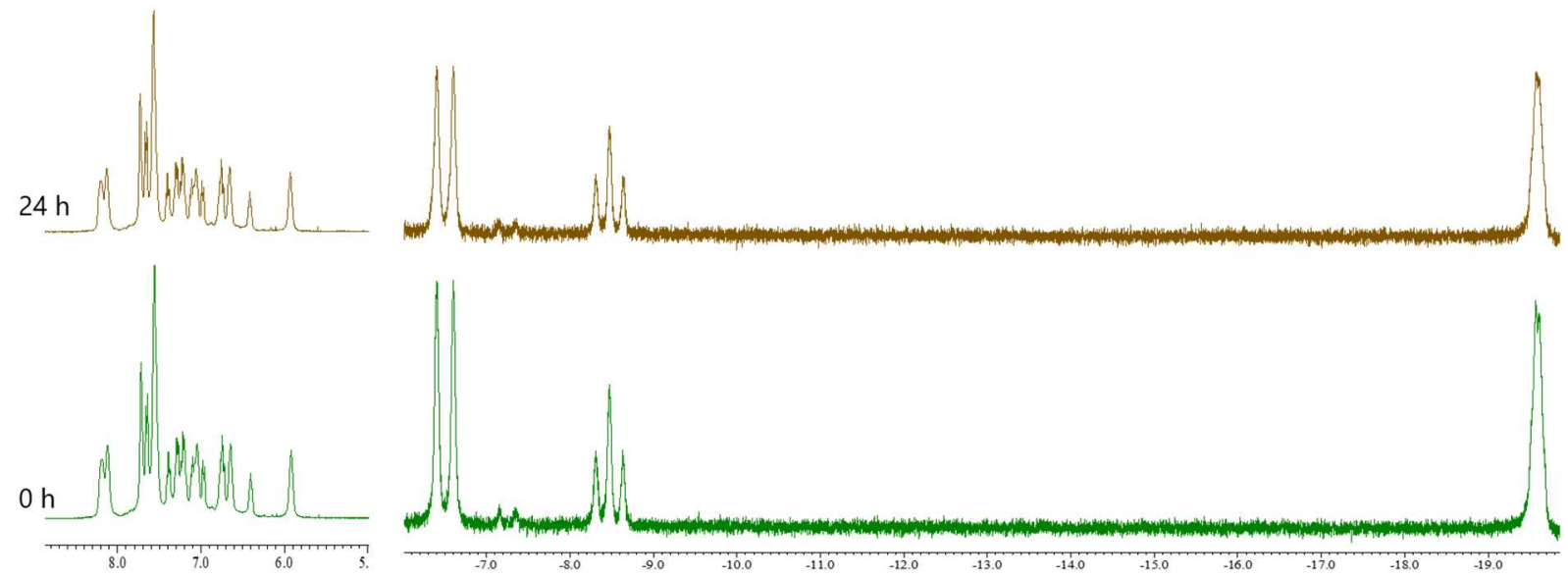

Figure S15. ${ }^{1} \mathrm{H}$ NMR spectra $\left(400 \mathrm{MHz}, \mathrm{rt}\right.$, acetone- $\left.d_{6}\right)$ of $\mathbf{2 a}$ in the H-D exchange reaction under dark condition.

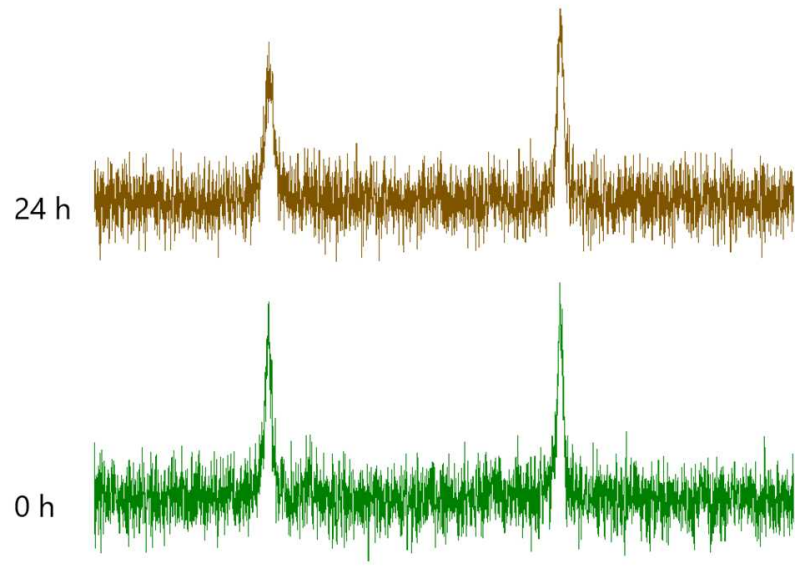

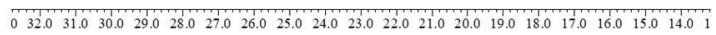

Figure S16. ${ }^{31} \mathrm{P}$ NMR spectra (162 MHz, rt, acetone- $d_{6}$ ) of 2a in the H-D exchange reaction under dark condition. 


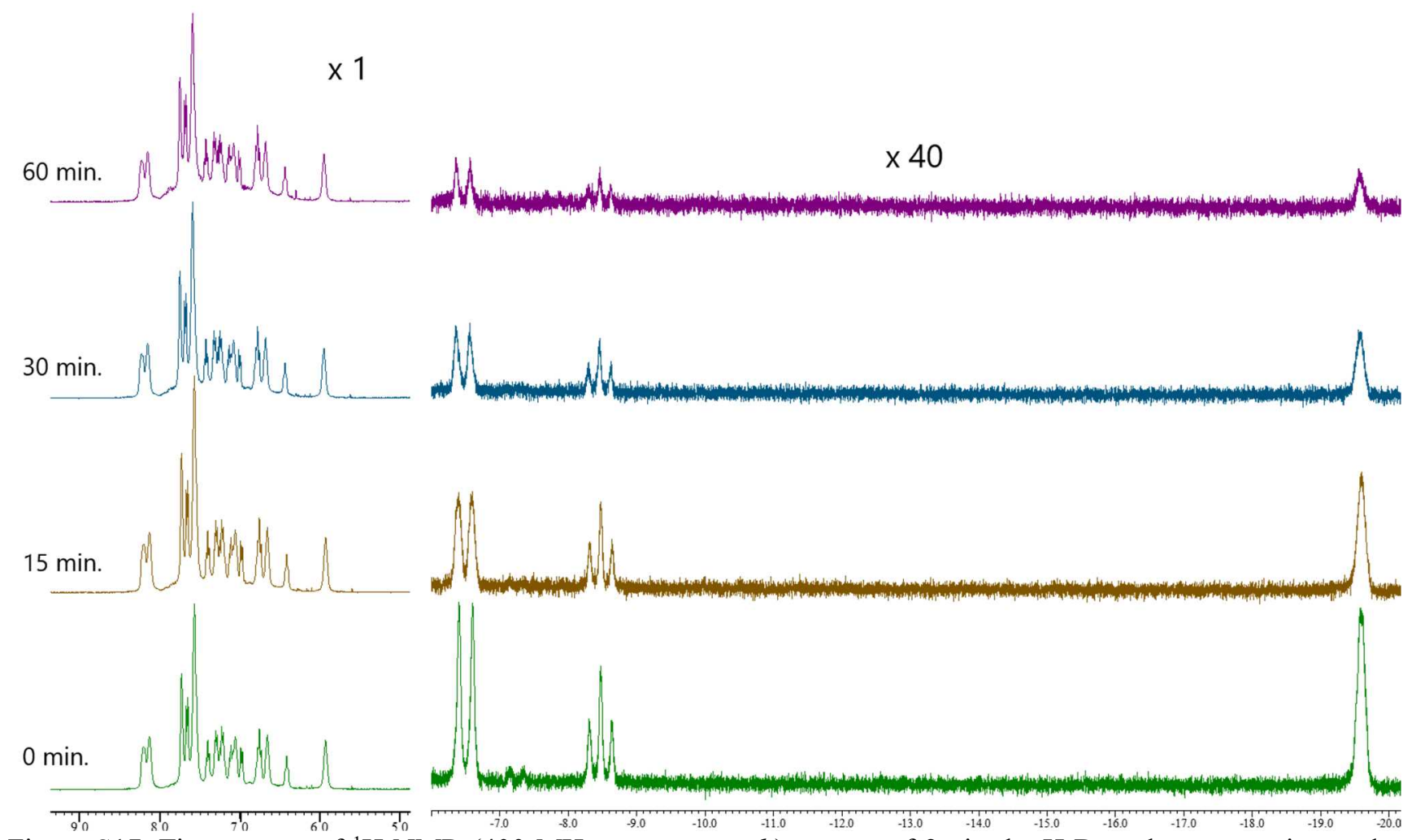

Figure S17. Time course of ${ }^{1} \mathrm{H}$ NMR (400 MHz, rt, acetone- $\left.d_{6}\right)$ spectra of $\mathbf{2 a}$ in the H-D exchange reaction under irradiation (395 nm).
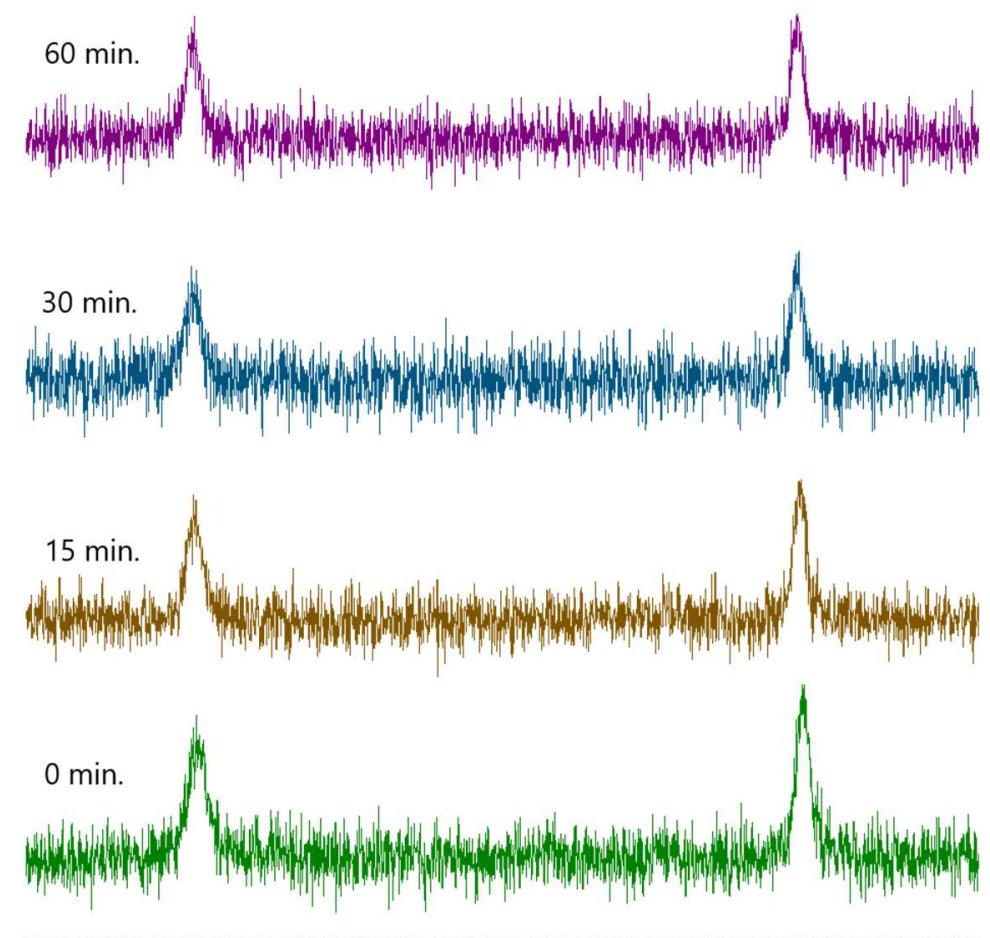

Figure S18. Time course of ${ }^{31} \mathrm{P}$ NMR spectra (162 MHz, rt, acetone- $\left.d_{6}\right)$ of $2 \mathbf{a}$ in the H-D exchange reaction under irradiation $(395 \mathrm{~nm})$. 


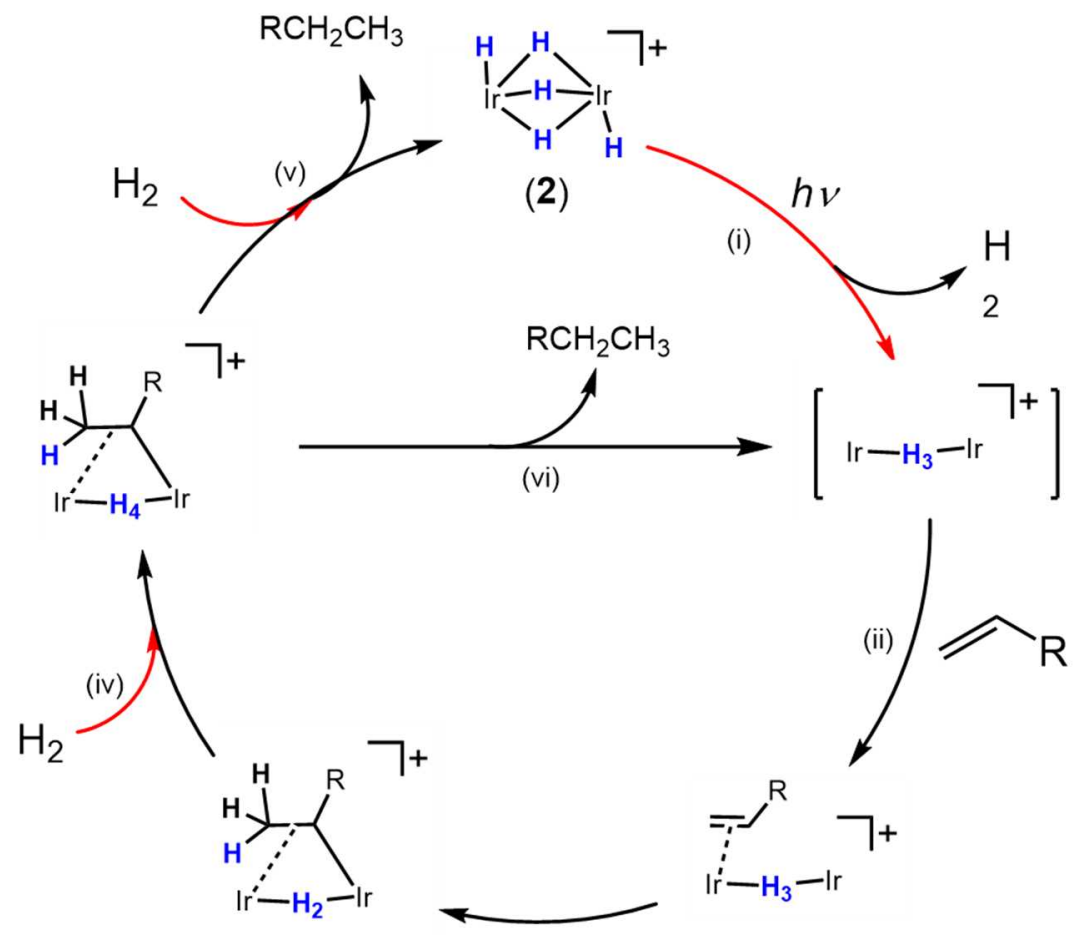

(iii)

Scheme S1. Plausible reaction mechanism of complex 2a-c with 1-hexene under photoirradiation. Path (i), (iv), and (v) are most probably accelerated by irradiation.

(i) Frisch, M. J.; Trucks, G. W.; Schlegel, H. B.; Scuseria, G. E.; Robb, M. A.; Cheeseman, J. R.; Scalmani, G.; Barone, V.; Petersson, G. A.; Nakatsuji, H.; Li, X.; Caricato, M.; Marenich, A. V.; Bloino, J.; Janesko, B. G.; Gomperts, R.; Mennucci, B.; Hratchian, H. P.; Ortiz, J. V.; Izmaylov, A. F.; Sonnenberg, J. L.; Williams-Young, D.; Ding, F.; Lipparini, F.; Egidi, F.; Goings, J.; Peng, B.; Petrone, A.; Henderson, T.; Ranasinghe, D.; Zakrzewski, V. G.; Gao, J.; Rega, N.; Zheng, G.; Liang, W.; Hada, M.; Ehara, M.; Toyota, K.; Fukuda, R.; Hasegawa, J.; Ishida, M.; Nakajima, T.; Honda, Y.; Kitao, O.; Nakai, H.; Vreven, T.; Throssell, K.; Montgomery, J. A., Jr.; Peralta, J. E.; Ogliaro, F.; Bearpark, M. J.; Heyd, J. J.; Brothers, E. N.; Kudin, K. N.; Staroverov, V. N.; Keith, T. A.; Kobayashi, R.; Normand, J.; Raghavachari, K.; Rendell, A. P.; Burant, J. C.; Iyengar, S. S.; Tomasi, J.; Cossi, M.; Millam, J. M.; Klene, M.; Adamo, C.; Cammi, R.; Ochterski, J. W.; Martin, R. L.; Morokuma, K.; Farkas, O.; Foresman, J. B.; Fox D. J. Gaussian 16, Revision A.03, Gaussian, Inc., Wallingford, CT, 2016.

(ii) (a) Becke, A. D. Density-Functional Exchange-Energy Approximation with Correct Asymptotic Behavior. Phys. Rev. A 1988, 38, 3098-3100; (b) Becke, A. D. Density-Functional Thermochemistry. III. The Role of Exact Exchange. J. Chem. Phys. 1993, 98, 5648-5652; (c) Lee, C.; Yang, W.; Parr, R. G. Development of the Colle-Salvetti Correlation-Energy Formula into a Functional of the Electron Density. Phys. Rev. B 1988, 37, 785-789.

(iii) (a) Dunning, T. H., Jr.; Hay, P. J. In Modern Theoretical Chemistry; Schaefer, H. F., III, Ed.; Plenum: New York, 1976; Vol. 3, pp 1-28; (b) Hay, P. J.; Wadt, W. R. Ab Initio Effective Core Potentials for Molecular Calculations. Potentials for the Transition Metal Atoms Sc to Hg. J. Chem. Phys. 1985, 82, 270-283; (c) Hay, P. J.; Wadt, W. R. Ab Initio Effective Core Potentials for Molecular Calculations. Potentials for $\mathrm{K}$ to Au Including the Outermost Core Orbitals. J. Chem. Phys. 1985, 82, 299-310; (d) Wadt, W. R.; Hay, P. J. Ab Initio Effective Core Potentials for 
Molecular Calculations. Potentials for Main Group Elements Na to Bi. J. Chem. Phys. 1985, 82, 284-298.

(iv) Petersson, G. A.; Bennett, A.; Tensfeldt, T. G.; Al-Laham, M. A.; Shirley, W. A.; Mantzaris, J. A Complete Basis Set Model Chemistry. I. The Total Energies of Closed-Shell Atoms and Hydrides of the First-Row Elements. J. Chem. Phys. 1988, 89, 2193-2218.

(v) Binkley, J. S.; Pople, J. A.; Hehre, W. J. Self-Consistent Molecular Orbital Methods. 21. Small Split-Valence Basis Sets for First-Row Elements. J. Am. Chem. Soc. 1980, 102, 939-947. 